\title{
Computational modeling of high performance steel fiber reinforced concrete using a micromorphic approach
}

\author{
A. E. Huespe · J. Oliver - D. F. Mora
}

Received: 16 January 2013 / Accepted: 1 May 2013

(C) Springer-Verlag Berlin Heidelberg 2013

\begin{abstract}
A finite element methodology for simulating the failure of high performance fiber reinforced concrete composites (HPFRC), with arbitrarily oriented short fibers, is presented. The composite material model is based on a micromorphic approach. Using the framework provided by this theory, the body configuration space is described through two kinematical descriptors. At the structural level, the displacement field represents the standard kinematical descriptor. Additionally, a morphological kinematical descriptor, the micromorphic field, is introduced. It describes the fibermatrix relative displacement, or slipping mechanism of the bond, observed at the mesoscale level. In the first part of this paper, we summarize the model formulation of the micromorphic approach presented in a previous work by the authors. In the second part, and as the main contribution of the paper, we address specific issues related to the numerical aspects involved in the computational implementation of the model. The developed numerical procedure is based on a mixed finite element technique. The number of dofs per node changes according with the number of fiber bundles simulated in the composite. Then, a specific solution scheme is proposed to solve the variable number of unknowns in the discrete model.
\end{abstract}

\section{A. E. Huespe $(\square)$}

CIMEC/CONICET-UNL, Güiemes 3450,

3000 Santa Fe, Argentina

e-mail: ahuespe@intec.unl.edu.ar

J. Oliver

E.T.S. Enginyers de Camins, Canals i Ports/CIMNE,

Technical University of Catalonia (UPC), Campus Nord

UPC (mòdul C-1), Jordi Girona 3, 08034 Barcelona, Spain

e-mail: xavier.oliver@upc.edu

D. F. Mora

IMDEA Materials, C/Eric Kandel 2, Tecnogetafe,

28906 Getafe, Spain

e-mail: diego.mora@imdea.org
The HPFRC composite model takes into account the important effects produced by concrete fracture. A procedure for simulating quasi-brittle fracture is introduced into the model and is described in the paper. The present numerical methodology is assessed by simulating a selected set of experimental tests which proves its viability and accuracy to capture a number of mechanical phenomenon interacting at the macro- and mesoscale and leading to failure of HPFRC composites.

Keywords High performance fiber reinforced concrete (HPFRC) - Failure of HPFRC - Short reinforcement fibers . Micromorphic materials - Material multifield theory . Morphological descriptors

\section{Introduction}

Cementitious materials such as mortar or concrete are brittle and have an inherent weakness in resisting tensile stresses. The addition of discontinuous fibers leads to a dramatic improvement in their toughness.

In conventional fiber reinforced concrete (conventional FRC), the fiber content is usually low and the tensile response is characterized by the opening of a single crack, similar to an unreinforced concrete [9]. While, high performance fiber reinforced cement composites (hereafter denoted as HPFRC composite) are highly ductile and characterized by pseudostrain hardening in tension. Consequently, strain hardening and multiple cracking constitute the main phenomenological differences between FRC and HPFRC composite.

Cement fracture is the mechanism that triggers the failure of HPFRC composites. However, the subsequent chain of events leading to the complete HPFRC failure is completely modified by the relative contents of fibers in the composite, and much more important, by the bond characteristic at the 
$$
71
$$

72 In the present work, we detail several issues related to 3 the numerical implementation and algorithmic aspects of the model that are specifically adopted for adequately solving large HPFRC composite problems with arbitrary directions of reinforcement fibers.

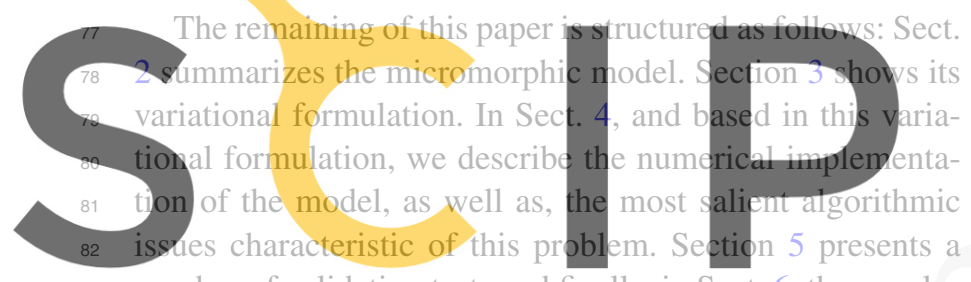
number of validation tests and finally, in Sect. 6, the conclu-

Register for free at https//WwW.scipedia.com to

85

86

${ }_{93} \quad \boldsymbol{x}=\hat{\boldsymbol{x}}(\boldsymbol{X}, t)$,

${ }_{98} \boldsymbol{\beta}=\hat{\boldsymbol{\beta}}(\boldsymbol{X}, t)$,

\section{Description of the HPFRC micromorphic model}

This section is devoted to summarize the HPFRC micromorphic model that has been presented in Oliver et al. [18].

\subsection{Deformation, morphological descriptor and strain} measures

The fundamentals of the model kinematical description are sketched in Fig. 1. We denote $\mathcal{B}_{0}$ the reference configuration of the body in the Euclidean space, and $\hat{\boldsymbol{x}}$ is the map:

specifying the current placement, of the particle $X$ in the body configuration at time $t$. In order to take into account the mesoscopic phenomena related to the sliding mechanisms of the fiber-matrix bond, we introduce a continuous microfield:

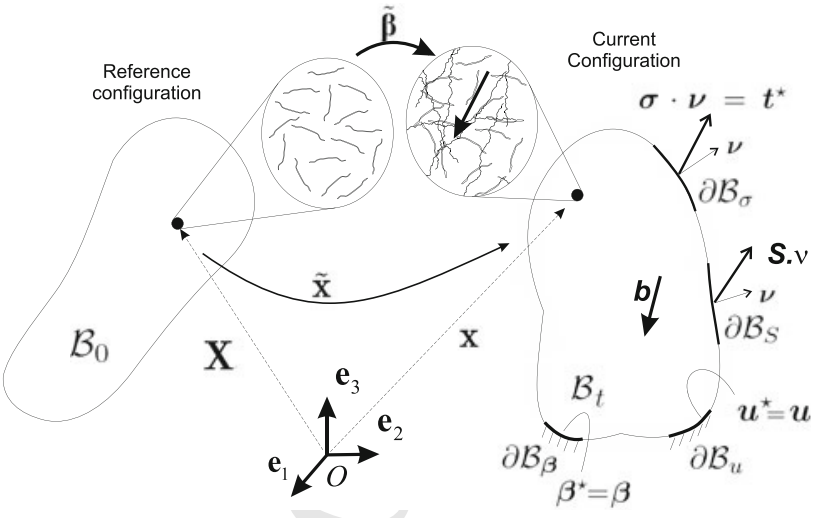

Fig. 1 Kinematical description of the HPFRC mechanical model

representing the relative displacement between the fiber and the matrix, as sketched in Fig. 1. According with the material multifield theory $[3,6,11], \beta$ can be thought as a substructural morphological descriptor.

Considering a local coordinate system ( $\boldsymbol{r} s$ ) where $\boldsymbol{r}$ is parallel to the fiber direction, see Fig. 2a, the relative fibermatrix displacement is supposed to have only one component, parallel to $r$, i.e. an axial component. Then, the sub-

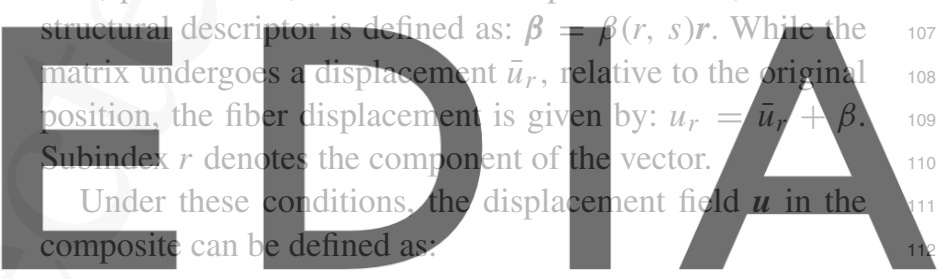

$u(\boldsymbol{X}, t)=\bar{u}(\boldsymbol{X}, t)+\mu_{f}(\boldsymbol{X}) \beta(\boldsymbol{X}, t)$

download the version without the watermark where $\mu_{f}$ is a spatial collocation function given by:

$\mu_{f}(\boldsymbol{X})= \begin{cases}0 & \text { if } \boldsymbol{X} \in \text { the concrete domain } \\ 1 & \text { if } \boldsymbol{X} \in \text { the fiber domain. }\end{cases}$

The displacement field (3) characterizing the composite deformation is sketched in Fig. 2. Figure $2 b$ shows the case when $\boldsymbol{\beta}=\boldsymbol{0}$ (i.e., the fiber is rigidly attached to the matrix), and Fig. 2c describes the case when $\boldsymbol{\beta} \neq \boldsymbol{O}$ (i.e., the fiber slides with respect to the matrix).

Considering plane problems in infinitesimal strains, the strain field can be expressed as:

$$
\begin{aligned}
\boldsymbol{\varepsilon}=\nabla^{s} \boldsymbol{u}= & \nabla^{s} \overline{\boldsymbol{u}}-\delta_{\Gamma} \boldsymbol{\beta}\left(\boldsymbol{r} \otimes^{s} \boldsymbol{s}\right) \\
& +\mu_{f}\left(\beta_{, r}(\boldsymbol{r} \otimes \boldsymbol{r})+\beta_{, s}\left(\boldsymbol{r} \otimes^{s} \boldsymbol{s}\right)\right),
\end{aligned}
$$

where the supra-index $(\cdot)^{s}$ denotes the symmetric open tensor product and subindices $(\cdot)_{, r}$ and $(\cdot)_{, s}$ denotes the derivatives respect to the coordinates $r$ and $s$, respectively. The second term in the right hand side is obtained after using the generalized gradient: $\nabla \mu_{f}=-\delta_{\Gamma} \boldsymbol{s}$, with $\delta_{\Gamma}$ being the Dirac's delta function shifted to the surface $\Gamma$ ( $\Gamma$ is the fiber-matrix interface shown in Fig. 2b). Thus, the overall strain $\varepsilon$ can be
116 117 118 119 120 121 122 123 124 125 126 127 128 129 130 131 


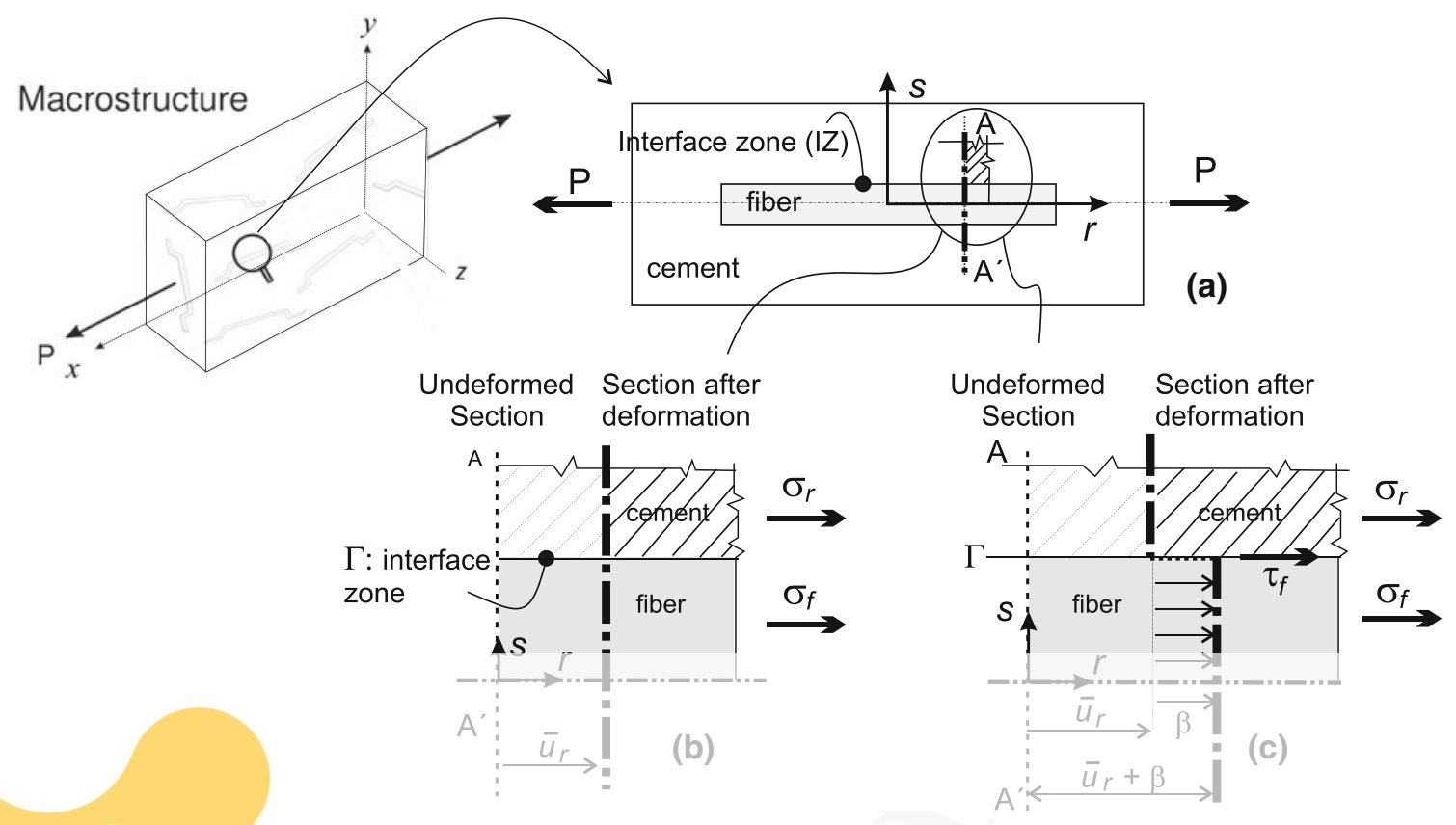

Fig. 2 HPFRC mechanical model at the mesostructural level. a The section $A-A^{\prime}$ of an undeformed unit cell, which includes a fiber with the surrounding concrete, moves to the position called "section after deformation" depending on whether the fiber-matrix interface remains rigidly attached (b) or slides (c)

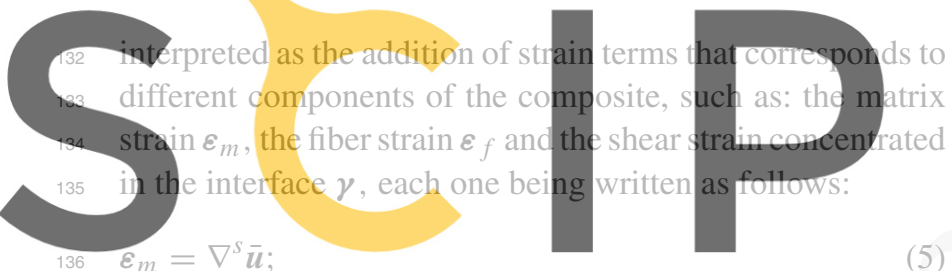

\section{$\varepsilon_{f}=\nabla^{s} \bar{u}+\left(\beta_{r}\left(r \otimes^{s} r\right)+\beta_{s}\left(r \otimes^{s} s\right)\right)$ \\ Register for free at https//www.scipedia.com to}

2.2 Generalized forces and balance equations: structural and substructural interactions

The momentum balance equations arising from the micromorphic material theory, see Mariano [11], are given by:

$\nabla \cdot \sigma+b=\boldsymbol{0} ; \quad \forall \boldsymbol{X} \in \mathcal{B}_{0}$

$\nabla \cdot S-z=\mathbf{0} ; \quad \forall X \in \mathcal{B}_{0}$,

with $\sigma$ being the conventional Cauchy stress tensor and $\boldsymbol{b}$ the body forces (per unit of volume) externally applied. Equation (8a) is the standard Cauchy equation, while (8b) represents the microscopic momentum balance equation provided by the multifield theory. The microstress $S$ is thermodynamically conjugate to $\nabla \dot{\beta}$, and $z$ are internal microforces, thermodynamically conjugate to $\dot{\boldsymbol{\beta}}$ which should necessarily exist to satisfy the framework invariance condition of the mechanical model (see Mariano and Stazi [12]). In this case, we have considered that any possible externally applied microforce is null.

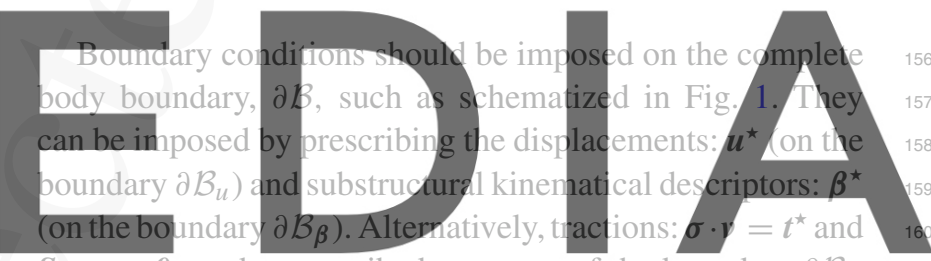

$S \cdot v=0$ can be prescribed on a part of the boundary $\partial \mathcal{B}_{\sigma}$ or $\partial \mathcal{B}_{S}$, respectively. Succh as happens in the conventional downtemos the versign awithout the pvatermark as: $\partial \mathcal{B}=\partial \mathcal{B}_{\beta} \cup \partial \mathcal{B}_{S}$ and $\varnothing=\partial \mathcal{B}_{\beta} \cap \partial \mathcal{B}_{S}$.

\subsection{HPFRC composite free energy}

The set of fibers oriented in an identical direction is here called a fiber bundle. First, let us consider a HPFRC composite having only one fiber bundle oriented in the direction provided by the constant unit vector $\boldsymbol{r}$. The free energy of the composite, $\psi$, is defined by adopting the mixture theory. By denoting $\psi_{m}, \psi_{f}$ and $\bar{\psi}_{\Gamma}$ the free energies of the matrix, fiber and interface components, respectively, and $k_{f}, k_{m}$ the volume fractions of the fiber and cement matrix, and such that: $k_{f}+k_{m}=1$; then, $\psi$ is defined as follows:

$$
\begin{aligned}
\psi\left(\nabla^{s} \overline{\boldsymbol{u}}, \boldsymbol{\beta}, \nabla \boldsymbol{\beta}, \boldsymbol{\alpha}\right)= & k_{m} \psi_{m}\left(\boldsymbol{\varepsilon}_{m}\left(\nabla^{s} \overline{\boldsymbol{u}}\right), r_{m}\right) \\
& +k_{f} \psi_{f}\left(\boldsymbol{\varepsilon}_{f}\left(\nabla^{s} \overline{\boldsymbol{u}}, \nabla \boldsymbol{\beta}\right), \alpha_{f}\right) \\
& +k_{f} \delta_{\Gamma} \bar{\psi}_{\Gamma}\left(\boldsymbol{\beta}, \alpha_{\Gamma}\right) ;
\end{aligned}
$$

where we have made explicit the dependence of $\psi$ with the kinematical variables, as well as, with the set of internal vari- 
${ }_{190} \quad S=\frac{\partial \psi}{\partial \nabla \beta}$

$$
z=\frac{\partial \psi}{\partial \beta},
$$

\section{where we identify $\boldsymbol{\sigma}_{m}$ and $\boldsymbol{\sigma}_{f}$ as the matrix and fiber stresses:}

\section{a $\psi$}

$\sigma_{m}=\frac{\partial \psi_{m}}{\partial \nabla^{s} \bar{u}} ; \quad \sigma_{f}=\frac{\partial \psi_{f}}{\partial \nabla^{s} \bar{u}}$
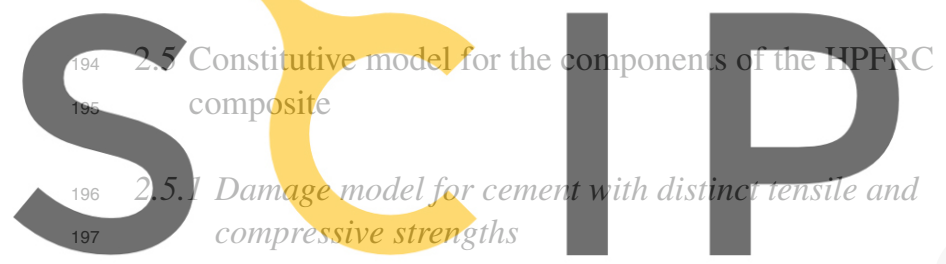

${ }_{198}$ The cementitious matrix is described using a standard Register for freegat hattps. th wistik. scipedia. compto

sive strengths. The equations describing the model are summanized in Table 1.

Equation (12) defines the free energy of this component. The term $d_{m}$ denotes the standard scalar damage variable. It is defined in Eq. (13) by introducing two additional internal variables: the stress-like internal variable $q_{m}$, which evolution equation is given in (19) in terms of the rate of the strainlike internal variable $r_{m}$ and the softening modulus $H_{m}<0$. The internal variable $r_{m}$ is defined in (16). The Hooke elastic tensor is denoted $\boldsymbol{C}_{m}$.

In Eq. (14), the stress-strain relation $\left(\boldsymbol{\sigma}_{m}\right.$ versus $\left.\boldsymbol{\varepsilon}_{m}\right)$ is given. The effective stress $\overline{\boldsymbol{\sigma}}_{m}$ is defined in Eq. (14b). Expressions (15) and (16), jointly with the complementarity conditions (20), defines the evolution equation for the internal variable $r_{m}$. Following the classical description of dissipative materials, $\lambda_{m}$ plays the role of a damage multiplier. The initial condition (16b) is given in terms of the ultimate tensile strength $\sigma_{m}^{u t}$ and the Young modulus $E_{m}$.

The damage criterion is defined in Eq. (17) where $\tau_{\varepsilon}$, defined in (18), represents a norm of the strains, with $\boldsymbol{C}_{m}$ working as a metric tensor. The functional dependence of

Table 1 Tensile-compressive concrete damage model

\section{Free energy}

$\psi_{m}\left(\boldsymbol{\varepsilon}_{m}\left(\nabla^{s} \overline{\boldsymbol{u}}\right), r_{m}\right)=\frac{1}{2}\left(1-d_{m}\right)\left(\boldsymbol{\varepsilon}_{m}: \boldsymbol{C}_{m}: \boldsymbol{\varepsilon}_{m}\right)$

Damage

$d_{m}=1-\frac{q_{m}\left(r_{m}\right)}{r_{m}}$

Stress-strain relationship

$\sigma_{m}=\frac{q_{m}}{r_{m}} \overline{\boldsymbol{\sigma}}_{m} ;$

where $\overline{\boldsymbol{\sigma}}_{m}=\boldsymbol{C}_{m}: \boldsymbol{\varepsilon}_{m}$

Internal variable evolution

$\dot{r}_{m}=\lambda_{m}$

$r_{m}=\max _{s \in[0, t]}\left[r_{0}, \tau_{\varepsilon}\left(\varepsilon_{m}(s)\right)\right] ;\left.\quad r_{m}\right|_{t=0}=r_{0}=\frac{\sigma_{m}^{u t}}{\sqrt{E_{m}}}$

Damage criterion

$f_{m}\left(\varepsilon_{m}, r_{m}\right)=\tau_{\varepsilon}-r_{m}$

$\tau_{\varepsilon}=\left(\theta+\frac{1-\theta}{n}\right) \sqrt{\bar{\sigma}_{m}:\left(\boldsymbol{C}_{m}\right)^{-1}: \bar{\sigma}_{m}}$.

$\theta=\frac{\sum_{i=1}^{3}\left\langle\bar{\sigma}_{m}^{i}\right\rangle}{\sum^{3}+\bar{\sigma}_{i} \mid}$

Stress-like internal variable and isotropic hardening law

$\dot{q}_{m}=H_{m}\left(r_{m}\right) \dot{r}_{m} ; \quad 0 \leq q_{m} \leq r_{0} ;\left.\quad q_{m}\right|_{t=0}=r_{0}$

Complementarity conditions

$f_{m} \leq 0 ; \quad \lambda_{m} \geq 0 ; \quad \lambda_{m} f_{m}=0$

Tangent constitutive operator

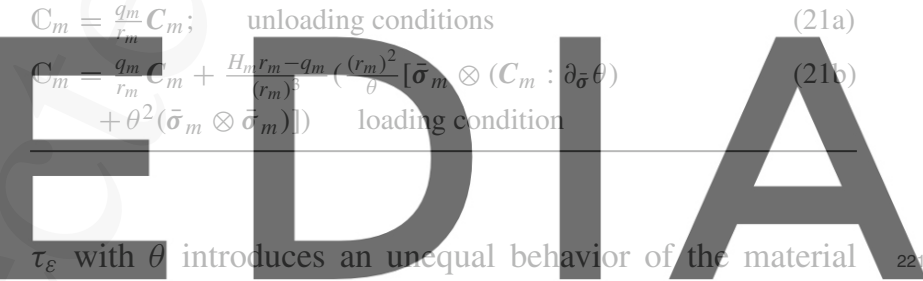

in tensile or compressive stress regimes. The ith princidow effective stress value is $\bar{\sigma}^{i}$ and the symbol $\langle\cdot\rangle$ denotes ${ }^{223}$ $\left(\sigma_{m}^{u c}\right)$ and tensile $\left(\sigma_{m}^{u t}\right)$ uniaxial ultimate strengths is denoted $n=\sigma_{m}^{u c} / \sigma_{m}^{u t}$. In the Haigh-Westergaard stress space, the trace of the damage criterion with the plane $\left(\bar{\sigma}_{m}^{1}, \bar{\sigma}_{m}^{2}\right)$ is shown in Fig. 3a. The interior points of the domain bounded by the trace represents the elastic domain. In the same figure (right), it is shown the possible evolution of an uniaxial loading/unloading process displaying distinct ultimate tensile and compressive strengths.

Equations (21) define the tangent constitutive tensors for both cases of the matrix damage evolutions: unloading or loading behavior. The term: $\partial_{\bar{\sigma}} \theta$, arising in (21b), has been described in [10], see also [16].

\subsubsection{One-dimensional elasto-plastic model for the fiber}

The fiber is characterized using a standard 1D plasticity model whose equations are summarized in Table 2.

Equation (23) defines the free energy of this component which is additively decomposed according with the mechanisms that activates the free energy change, i.e. elastic or plastic processes. Also, the fiber strain $\boldsymbol{\varepsilon}_{f}$ is assumed to be

\section{5}

226 227 228 229 230 231 232 233 234 235 236 


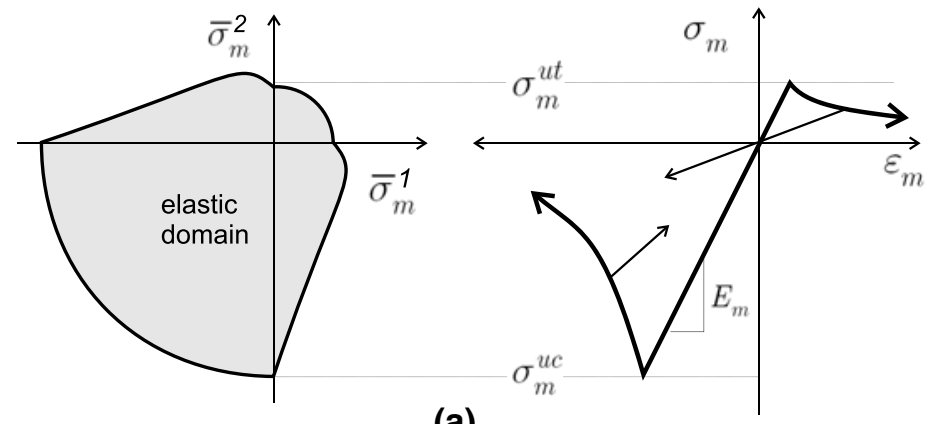

(a)

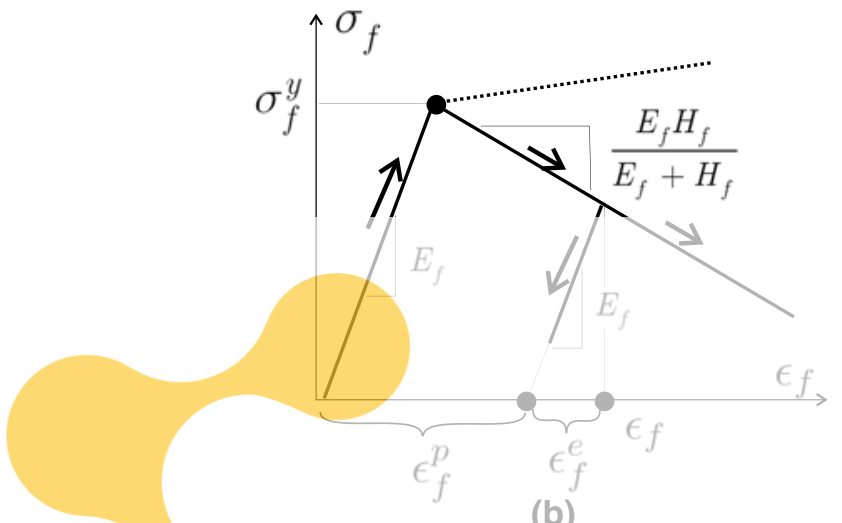

(b)

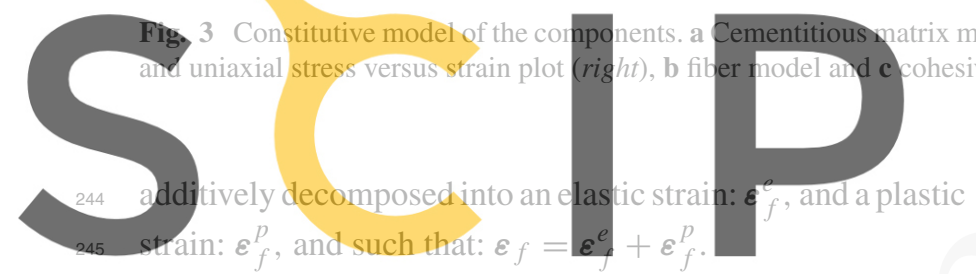

The elastic constitutive tensor is denoted $\mathbb{E}_{f}$, and has only

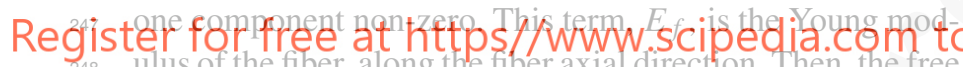
ulus of the fiber, along the fiber axial direction. Then, the free

49 energy can be written in terms of the axial component of the

250

251 (elastic) fiber strain:

$\epsilon_{f}=\boldsymbol{\varepsilon}_{f}:(\boldsymbol{r} \otimes \boldsymbol{r})$,

with $\epsilon_{f}=\left(\bar{u}_{r}\right)_{, r}+\beta_{, r}$, where we have also considered the additive decompositions of the uniaxial fiber strains: $\epsilon_{f}=$ $\epsilon_{f}^{e}+\epsilon_{f}^{p}$.

The internal variable $\alpha_{f}$ represents the equivalent plastic strain of the fiber. Then, $\psi_{f}^{h}$ represents the energetic hardening.

The remaining equations are standard, and closely follow the models presented in classical books of plasticity, see for example [20]. The only stress component that is significant in this elasto-plastic model is the axial stress: $\sigma_{f}=\sigma_{f}:(\boldsymbol{r} \otimes \boldsymbol{r})$ and is defined in Eq. (24) in terms of the fiber elastic axial strain.

Note that the material parameters defining the model are: the Young modulus $E_{f}$, the softening/hardening modulus $H_{f}$ and the yield stress $\sigma_{f}^{y}$. Figure $3 \mathrm{~b}$ depicts a typical stressstrain response of this model.

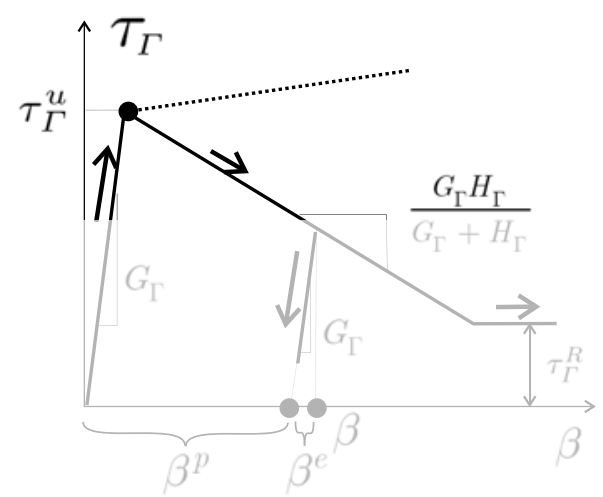

(c)

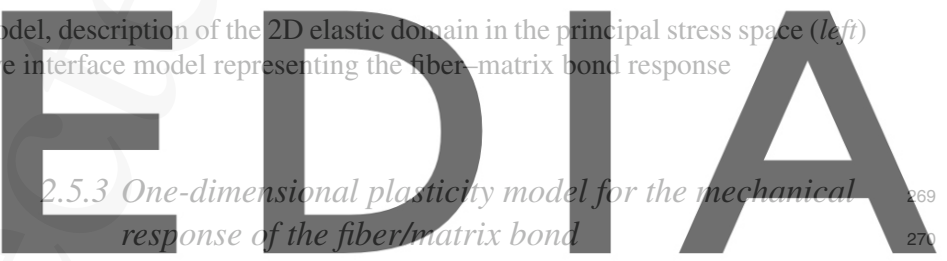
component) is assumed to be additively decomposed into an $\quad 275$ elastic part $\beta^{e}$ and a plastic part $\beta^{p}$, respectively.

The equations are summarized in Table 3 which interpretation are similar to that exposed in Table 2.

In Eq. (31), we define a specific (per unit of area) free energy $\bar{\psi}_{\Gamma}$. The term $G_{\Gamma}$ is a pseudo-shear modulus that is sufficiently large to avoid large fiber-matrix slips $\beta^{e}$ with shear stresses $\tau_{\Gamma}<\tau_{\Gamma}^{u}$.

The material parameters in this case are: the elastic shear modulus $G_{\Gamma} \mathrm{N} / \mathrm{m}^{3}$ ) the ultimate bond strength $\tau_{\Gamma}^{u}$, the softening/hardening modulus $H_{\Gamma}$ and the residual bond strength $\tau_{\Gamma}^{R}$. A typical response of the fiber-matrix bond model is depicted in Fig. 3c.

From Eq. (11) and Tables 1 and 2, the matrix and fiber stresses can be expressed as: 
Table 2 Fiber 1D elasto-plastic model

Free energy

$\psi_{f}\left(\boldsymbol{\varepsilon}_{f}\left(\nabla^{s} \overline{\boldsymbol{u}}, \nabla \beta\right), \alpha_{f}\right)=\frac{1}{2}\left(\boldsymbol{\varepsilon}_{f}^{e}: \mathbb{E}_{f}: \boldsymbol{\varepsilon}_{f}^{e}\right)+\psi_{f}^{h}\left(\alpha_{f}\right)$

$$
=\frac{1}{2} E_{f}\left[\epsilon_{f}^{e}\right]^{2}+\psi_{f}^{h}\left(\alpha_{f}\right)
$$

$\mathbb{E}_{f}=E_{f}(\boldsymbol{r} \otimes \boldsymbol{r}) \otimes(\boldsymbol{r} \otimes \boldsymbol{r}) ; \quad \boldsymbol{\varepsilon}_{f}=\boldsymbol{\varepsilon}_{f}^{e}+\boldsymbol{\varepsilon}_{f}^{p}$

Elastic stress-strain relationship

$\sigma_{f}=E_{f} \epsilon_{f}^{e}$ with $\epsilon_{f}=\left(\bar{u}_{r}\right)_{, r}+\beta_{, r}$

Flow rule

$\epsilon_{f}^{p}=\lambda_{f} \operatorname{sign}\left(\sigma_{f}\right)$

Internal variable evolution

$\dot{\alpha}_{f}=\lambda_{f}$

Isotropic hardening law

$\dot{q}_{f}=H_{f}\left(\alpha_{f}\right) \dot{\alpha}_{f}$

Yield function

$f_{f}=\left|\sigma_{f}\right|-\left(q_{f}+\sigma_{f}^{y}\right)$

Complementarity conditions

$f_{f} \leq 0 ; \quad \lambda_{f} \geq 0 ; \quad \lambda_{f} f_{f}=0$

Tangent constitutive operator

$\mathbb{C}_{f}=C_{f}[(\boldsymbol{r} \otimes r) \otimes(r \otimes r)]$

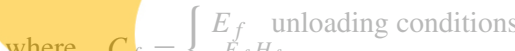

where $\quad C_{f}= \begin{cases}\frac{E_{f} H_{f}}{E_{f}+H_{f}} ; & \text { loading conditions }\end{cases}$

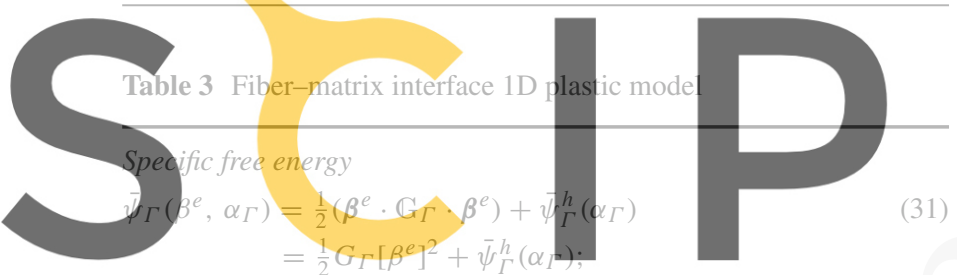

$\mathbb{G}_{\Gamma}=G_{\Gamma}(r \otimes r) ; \quad \beta=\beta^{e}+\beta^{P}$

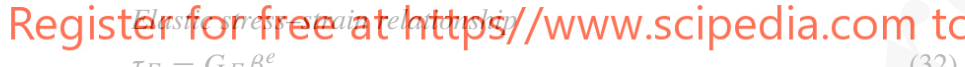

$\tau_{\Gamma}=G_{\Gamma} \beta^{e}$

Flow rule

$\dot{\beta}^{p}=\lambda_{\Gamma} \operatorname{sign}\left(\tau_{f}\right)$

Internal variable evolution

$\dot{\alpha}_{\Gamma}=\lambda_{\Gamma}$

Yield function

$f_{\Gamma}\left(\tau_{\Gamma}, \alpha_{\Gamma}\right)=\left|\tau_{\Gamma}\right|-\left(q_{\Gamma}+\tau_{\Gamma}^{u}\right)$

Isotropic hardening law

$\dot{q}_{\Gamma}=H_{\Gamma}\left(\alpha_{\Gamma}\right) \dot{\alpha}_{\Gamma}$

Complementarity conditions

$f_{\Gamma} \leq 0 ; \quad \lambda_{\Gamma} \geq 0 ; \quad \lambda_{\Gamma} f_{\Gamma}=0$

Tangent constitutive operator

$C_{\Gamma}=G_{\Gamma} ;$ unloading condition

$C_{\Gamma}=\frac{G_{\Gamma} H_{\Gamma}}{G_{\Gamma}+H_{\Gamma}} ;$ loading condition

${ }_{292}^{291} \quad \boldsymbol{\sigma}_{m}=\frac{\partial \psi_{m}}{\partial \nabla^{s} \overline{\boldsymbol{u}}}=\left(1-d_{m}\right) \boldsymbol{C}_{m}: \boldsymbol{\varepsilon}_{m}$

$\boldsymbol{\sigma}_{f}=\frac{\partial \psi_{f}}{\partial \nabla^{s} \overline{\boldsymbol{u}}}=\sigma_{f}(\boldsymbol{r} \otimes \boldsymbol{r})$

The microstress $\boldsymbol{S}$ are given by:

$\boldsymbol{S}=\frac{\partial \psi}{\partial \nabla \beta}=\mu_{f} k_{f} \sigma_{f}(\boldsymbol{r} \otimes \boldsymbol{r})$

296

and the microforce $z$ is:

$z=\frac{\partial \psi}{\partial \beta}=-\delta_{\Gamma}\left(k_{f} \tau_{\Gamma}\right) \boldsymbol{r}$

Summarizing, the stresses of the different components can be written as follows:

(i) matrix stress (given in Table 1):

303

$\sigma_{m}=\hat{\sigma}_{m}\left(\varepsilon_{m}\left(\nabla^{s} \overline{\boldsymbol{u}}, \boldsymbol{r}_{m}\right)\right)$

(ii) fiber stress (Table 2):

$\sigma_{f}=\hat{\sigma}_{f}\left(\epsilon_{f}\left(\left(\bar{u}_{r}\right)_{, r}, \beta_{, r}\right), \alpha_{f}\right)$,

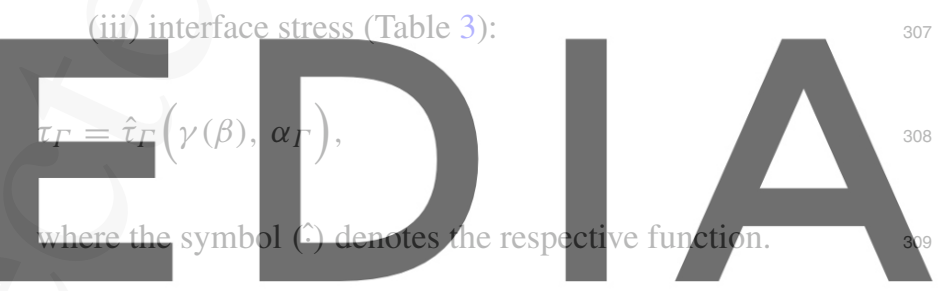

2.7 The overall constitutive model of a HPFRC composite

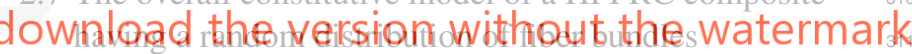

The mechanical model of a HPRFC having a fiber bundle in one direction, presented in the previous subsections, can be generalized to account for a random distribution of fibers. Let us consider a number $n_{f}$ of discrete fiber bundles in the plane of analysis with a regular distribution of angles in the interval: $[0, \pi]$.

The Ith bundle, characterized with the supra-index $I$, $\left(I=1, \ldots, n_{f}\right)$, has assigned one volume fraction $k_{f}^{I}$, one direction vector $\boldsymbol{r}^{I}$ and one micromorphic field $\boldsymbol{\beta}^{I}=\beta^{(I)} \boldsymbol{r}^{(I)}$ (supra-index in parenthesis indicates no-summation on this index).

Adopting the mixture theory of Truesdell to account for the macro/mesoscopic interactions, the free energy of the HPFRC composite can be written as the linear combination of the free energies of all its components, weighted by the corresponding volume fraction:

312

313

314

$\psi=k_{m} \psi_{m}+\sum_{I=1}^{n_{f}} k_{f}^{I} \psi_{f}^{I}+\sum_{I=1}^{n_{f}} k_{f}^{I} \bar{\psi}_{\Gamma}^{I}$. 


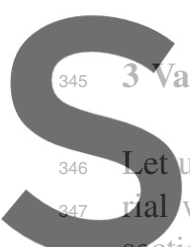

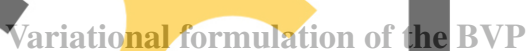

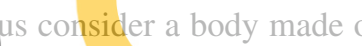
which is modeled such as de

\section{HPFR C} scribed in the preceding
of the RVP are: (i) the displacement-strain equations (3), (5)-(7), (ii) the consti-

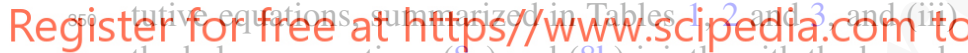
the balance equations (8a) and (8b) jointly with the boundary conditions. In the complete boundary of the body $\partial \mathcal{B}$, we

Table 4 Variational BVP

$$
\begin{gathered}
\int_{\mathcal{B}} \sigma: \nabla^{s} \delta \overline{\boldsymbol{u}} d \mathcal{B}-\int_{\mathcal{B}} \boldsymbol{b} \cdot \delta \overline{\boldsymbol{u}} d \mathcal{B}-\int_{\partial \mathcal{B}_{\sigma}} t^{*} \cdot \delta \overline{\boldsymbol{u}} d S=0 ; \\
\forall \delta \overline{\boldsymbol{u}} \in \mathcal{V}_{0} \\
\int_{\mathcal{B}}\left(\frac{\Pi^{I}}{A^{I}} \tilde{\tau}_{f}^{I} \delta \beta^{I}+\tilde{\sigma}_{f}^{I}\left(\delta \beta^{I}\right)_{, r}\right) d \mathcal{B}=0 ; \\
\forall \delta \beta^{(I)} \in \mathcal{V}_{0}^{\beta} ; \quad\left(I=1, \ldots, n_{f}\right)
\end{gathered}
$$

equation (8b). In Eq. (48), $\Pi^{I}$ and $A^{I}$ are the perimeter 370 and area of one representative fiber of the fiber bundle $I, \quad 371$ respectively. Both terms arise as a result of the averaging $\quad 372$ process of the stresses in the fibers.

(ii) we consider identical models to those presented in Tables 2 and 3, to express the constitutive response of the averaged stresses $\tilde{\sigma}_{f}$ and $\tilde{\tau}_{\Gamma}$ in terms of the averaged strain quantities: $\tilde{\epsilon}_{f}$ and $\tilde{\gamma}$; and the model in Table 1 .

Note that both expressions (47) and (48), in Table 4, have been derived by weakening the derivative of the stress terms and imposing the boundary conditions in the boundary integrals.

Additional details of the variational BVP equations can be seen in Oliver et al. [18].

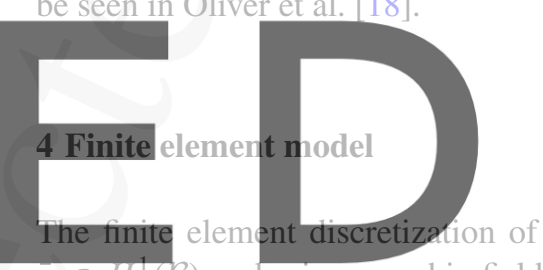

$u \in H^{1}(\mathcal{B})$ and micromorphic field $\beta$

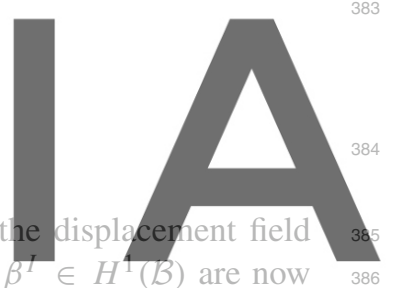

considered. Both of them are interpolated using a standard 387 downdQaentthenversion without the watermarks

$$
\begin{aligned}
\overline{\boldsymbol{u}}(\boldsymbol{x}, t) & =\sum_{j=1}^{n_{\text {node }}} N_{j}(\boldsymbol{x}) \boldsymbol{q}_{j}(t) ; \\
\beta^{I}(\boldsymbol{x}, t) & =\sum_{j=1}^{n_{\text {node }}} N_{j}(\boldsymbol{x}) p_{j}^{I}(t) ;
\end{aligned}
$$

where $N_{j}$ are the shape functions of the finite element and $\boldsymbol{q}_{j}$ and $p_{j}^{I}$ are the displacement vector and the $I$ th micromorphic descriptor of the node $j$, respectively. The total number of nodes in the finite element mesh is denoted $n_{\text {node }}$. While, the corresponding variations are given:

$\delta \boldsymbol{u}(\boldsymbol{x}, t)=\sum_{j=1}^{n_{\text {node }}} N_{j}(\boldsymbol{x}) \delta \boldsymbol{q}_{j}(t) ;$

(i) we evaluate the average stress $\tilde{\sigma}_{f}$ (of the term $\sigma_{f}$ ) in the cross section of the fiber, and the average shear stress $\tilde{\tau}_{\Gamma}$ (of the term $\tau_{\Gamma}$ ) along the fiber circumferential perimeter. Then, we introduce both average stresses into the balance $\delta \beta^{I}(\boldsymbol{x}, t)=\sum_{j=1}^{n_{\text {node }}} N_{j}(\boldsymbol{x}) \delta p_{j}^{I}(t)$.
393 394 395 396 397 
Re ${ }^{422}$ and $\left[N_{r}\right]_{r}\left[N_{s}\right]$ are the $r$ and $s$ derivatives of the shape funcRegister for free at https//www.scipedia.com to

424 with $n_{\text {node }}^{e}$ being the number of nodes in element $e$, and the nodal displacement vectors of the same element $e$ is denoted $q^{e}$. From Eq. (6), the fiber strain vector, of the fiber bundle $I$, is:

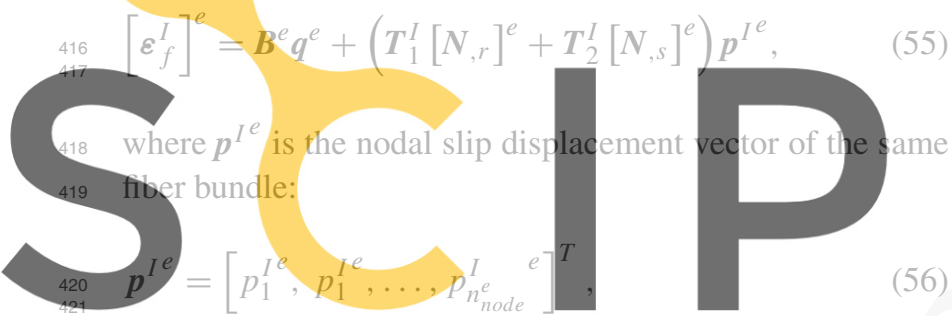

Using Eq. (5), the interpolated strain terms in the finite element $e$ can be written as follows:

$\left[\boldsymbol{\varepsilon}_{m}\right]^{e}=\left[\nabla^{s} \overline{\boldsymbol{u}}\right]^{e}=\boldsymbol{B}^{e} \boldsymbol{q}^{e}$,

where we have used the symbol $[\cdot]$ to represent the vector Voigt notation of the corresponding tensor. The standard strain-displacement matrix $\boldsymbol{B}^{e}$ of the element $e$ is:

$\boldsymbol{B}^{e}=\left[\boldsymbol{B}_{1}^{e}, \boldsymbol{B}_{2}^{e}, \ldots, \boldsymbol{B}_{n_{\text {node }}^{e}}^{e}\right]$

$\boldsymbol{B}_{j}^{e}=\left[\begin{array}{cc}\left(N_{j}^{e}\right)_{, x} & 0 \\ 0 & \left(N_{j}^{e}\right)_{, y} \\ \left(N_{j}^{e}\right)_{, y} & \left(N_{j}^{e}\right)_{, x}\end{array}\right]$,

(56)
$\left[N_{, s}\right]=\left[\left(N_{1}\right)_{, s}, \ldots,\left(N_{n_{\text {node }}^{e}}\right)_{, s}\right]$,

where considering $N_{j}(\boldsymbol{x})$, then: $\left(N_{j}\right)_{, r}=\left(N_{j}\right)_{, x} x_{, r}+$ $\left(N_{j}\right)_{, y} y_{, r}$. Also, matrices $\boldsymbol{T}_{1}^{I}$ and $\boldsymbol{T}_{2}^{I}$ in Eq. (55), are the Voigt vector notation of the tensors: $\left(\boldsymbol{r}^{I} \otimes \boldsymbol{r}^{I}\right)$ and $\left(\boldsymbol{r}^{I} \otimes^{S} \boldsymbol{s}^{I}\right)$, respectively:

$\boldsymbol{T}_{1}^{I}=\left[r_{x}^{2}, r_{y}^{2}, 2 r_{x} r_{y}\right]_{I}^{T}$

$\boldsymbol{T}_{2}^{I}=\left[r_{x} s_{x}, r_{x} r_{y},\left(r_{x} s_{y}+r_{y} s_{x}\right)\right]_{I}^{T}$

The axial component of the fiber strain $I$ th can be written as follows:

$$
\left[\epsilon_{f}^{I}\right]^{e}=\left(\hat{\boldsymbol{T}}_{1}^{I}\right)^{T}\left[\boldsymbol{\varepsilon}_{f}^{I}\right]^{e}=\left(\hat{\boldsymbol{T}}_{1}^{I}\right)^{T} \boldsymbol{B}^{e} \boldsymbol{q}^{e}+\left[\boldsymbol{N}_{, r}\right]^{e}\left[\boldsymbol{p}^{I}\right]^{e},
$$

where the projection operator: $\hat{\boldsymbol{T}}_{1}^{I}$ is:

$\hat{\boldsymbol{T}}_{1}^{I}=\left[r_{x}^{2}, r_{y}^{2}, r_{x} r_{y}\right]$,

which satisfies: $\left(\hat{\boldsymbol{T}}_{1}^{I}\right)^{T} \boldsymbol{T}_{1}^{I}=1$ and $\left(\hat{\boldsymbol{T}}_{1}^{I}\right)^{T} \boldsymbol{T}_{2}^{I}=0$.

Finally, from Eq. (7), the strain vector representing the $I$ th fiber-matrix slip mechanisms, is written:

$\left[\boldsymbol{\gamma}^{I}\right]=\boldsymbol{T}_{2}^{I}[N]^{e} \boldsymbol{p}^{I^{e}}$

After introducing the finite element discretization into the balance equations (47), (48) jointly with the constitutive relations in Tables 1,2 and 3; the balance equations can be rewritten as a system of equations in the variables $\boldsymbol{q}, \boldsymbol{p}^{I}$ :
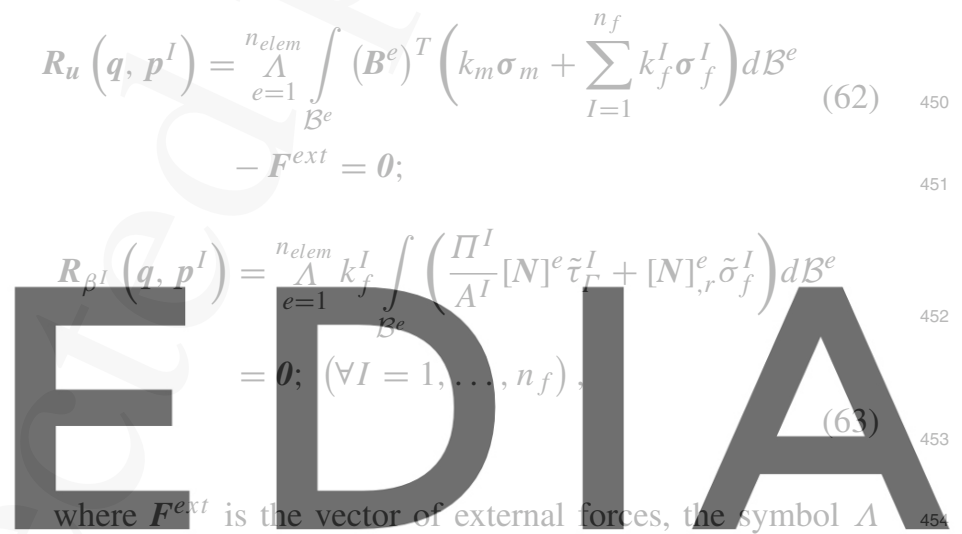

denotes the standard finite element assemblage operator, 455

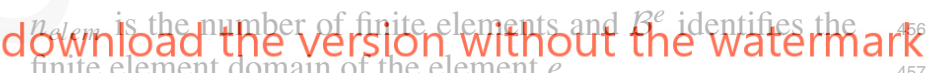
finite element domain of the element $e$.

\subsection{Time integration scheme}

The time integration problem consists of finding, at the time step $n+1$, the nodal displacements, $\boldsymbol{q}_{n+1}$, and micromorphic descriptors, $p_{n+1}^{I}$, verifying the equations of the discrete variational BVP (62), (63). We denote $\boldsymbol{p}_{n+1}$ the vector collecting the slips $\boldsymbol{p}_{n+1}^{I}$ of all fiber bundles. In those expressions, the stresses: $\sigma_{m}, \sigma_{f}^{I}$ and $\tilde{\tau}_{\Gamma}^{I}$ are explicit functions of $\left(\boldsymbol{q}_{n+1}, \boldsymbol{p}_{n+1}\right) . \boldsymbol{F}^{e x t}$ is evaluated at time $(n+1)$.

\subsubsection{Solution of the coupled system of equations}

Two general strategies can be adopted for solving the coupled problem (62), (63): monolithic and fractional step methods (also known as staggered techniques). The following items describe both strategies. 


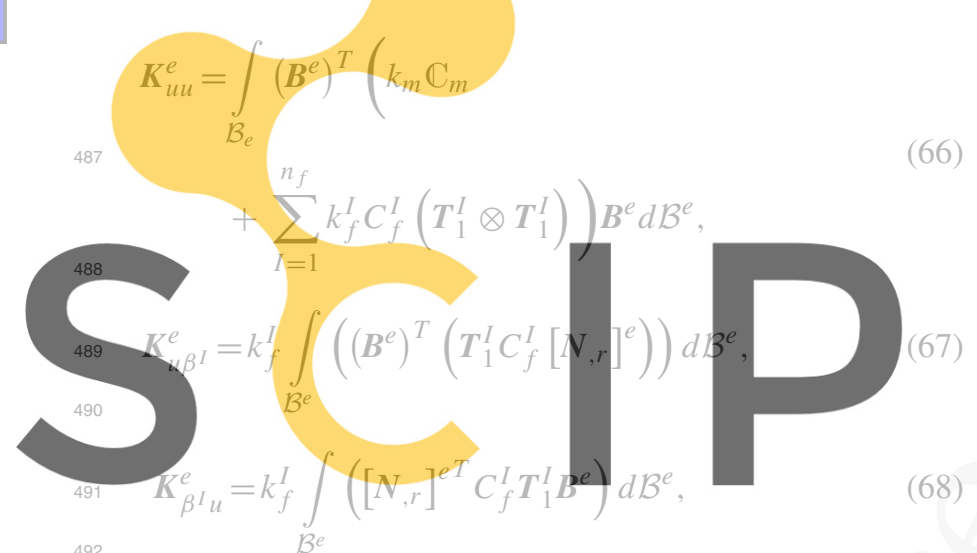

Register for free at https//www.scipedia.com to ${ }_{493} K_{\beta^{I} \beta^{I}}^{e}=k_{f}^{I} \int_{\mathcal{B}^{e}}\left(\frac{\Pi^{I}}{A^{I}}[N]^{e T} C_{\Gamma}^{I}[N]^{e}+\left[N_{, r}\right]^{e T} C_{f}^{I}\left[N_{, r}\right]^{e}\right) d \mathcal{B}^{e}$,

which consists of, iteratively and simultaneously, determining the increment of variables: $(\Delta \boldsymbol{q} ; \Delta \boldsymbol{p})$ by solving the linearized equation system derived from (62), (63):

$\boldsymbol{K}\left[\begin{array}{c}\Delta \boldsymbol{q} \\ \Delta \boldsymbol{p}^{I}\end{array}\right]=-\left[\begin{array}{c}\boldsymbol{R}_{u} \\ \boldsymbol{R}_{\beta^{I}}\end{array}\right]$

where $\boldsymbol{K}$ is the Jacobian of the residuals (62), (63):

$\boldsymbol{K}=\frac{\partial\left(\left[\boldsymbol{R}_{\boldsymbol{u}} ; \boldsymbol{R}_{\beta^{I}}\right]\right)}{\partial\left(\left[\boldsymbol{q} ; \boldsymbol{p}^{I}\right]\right)}=\stackrel{\Lambda_{e=1}^{n_{\text {elem }}}}{\Lambda_{\beta^{I} u}}\left[\begin{array}{cc}K_{u u}^{e} & K_{u \beta^{I}}^{e} \\ K_{\beta^{I}}^{e} & K^{e}\end{array}\right]$.

The expression for $\boldsymbol{K}$ is obtained by introducing the strains (53), (59), (61) into the constitutive Tables 1, 2 and 3, deriving the corresponding stresses and then, introducing them into the derivatives of the residual terms defined in (62), (63). Following this procedure, every submatrix in (65) can be vritten as follows:

where $\mathbb{C}_{m}$ is the matrix constitutive tangent tensors defined in Eq. (21). And, $C_{f}^{I}$ and $C_{\Gamma}^{I}$ are the constitutive tangent tensor of every fiber bundle defined in (30) and (38), respectively.

In order to preserve the notation as simple as possible, we do not specify the fact that, at step $n+1$, expressions $\boldsymbol{K}$ and $\boldsymbol{R}$ in (64) are evaluated in every iteration $k$ of the NewtonRaphson procedure.

(ii) Staggered scheme In the second procedure, and taking advantage of the physical nature of the problem, the equation system (62), (63) is partitioned into smaller and simpler subsystems. The solution of each subsystem determines one set of variables at a time, keeping fixed the remaining ones.

For this specific problem, a natural partition consists of taking as many set of equations as families of fiber bundles exists: $\boldsymbol{R}_{\beta^{I}}=\mathbf{0}$, for: $I=1, \ldots, n_{f}$ plus the equation of: $\boldsymbol{R}_{u}=0$.
Then, given a prediction of the slip field $\left(\boldsymbol{p}^{I}\right)_{n+1}^{P}$, which are the linear extrapolations of values obtained in previous time steps:

$$
\left(p_{n+1}^{I}\right)^{P}=\boldsymbol{p}_{n}^{I}+\left(\boldsymbol{p}_{n}^{I}-\boldsymbol{p}_{n-1}^{I}\right) \frac{\Delta t_{n+1}}{\Delta t_{n}},
$$

where $\Delta t_{n}$ and $\Delta t_{n+1}$ are the time increments in steps $n$ and $n+1$, respectively; the equation system:

$\boldsymbol{R}_{u}\left(\boldsymbol{q}_{n+1},\left(p_{n+1}^{I}\right)^{P}\right)=\mathbf{0}$,

is solved to find: $\boldsymbol{q}_{n+1}$. And this value is substituted, and fixed, in each set of Eq. (63):

$\mathbb{R}_{\beta^{I}}\left(q_{n+1}, p_{n+1}^{I}\right)=0$,

which solution provides the slip values $\boldsymbol{p}_{n+1}^{I}$

After replacing $\left(\boldsymbol{p}_{n+1}^{I}\right)^{P}$ by $\boldsymbol{p}_{n+1}^{I}$ in Eq. (71), the sequence of operations to solve (71) and (72) are repeated iteratively until obtaining the convergence of the equation system (62), (63) at time step: $n+1$.

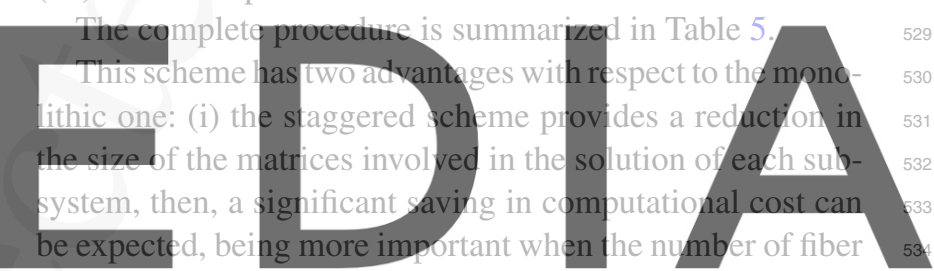

bundles increase; and (ii) the computational treatment (handling of dofs) pf problems with a yariable number of fiber

Prediction (70) has shown to be successful to increase the accuracy of the scheme. This effect can be seen in Fig. 4 that represents the structural response of the beam in Sect. 5.2 when the effect of the interface zone vanishes $\left(\tau_{\Gamma}^{u}=0\right)$. The plots depicted in the figure are the load versus vertical displacement of the load application point. Two solutions were obtained with a staggered integration scheme using either: (i) the extrapolation defined in (70), or (ii) without including the extrapolation. Both curves have been evaluated using the algorithm in Table 5 by removing the iterative procedure (loop on the index $k$ in the table). Thus, Eqs. (74) and (75) have been evaluated only once per time step. In the last case, when the predictor equation (73) is removed from the algorithm, $\left(\boldsymbol{p}_{n+1}^{I}\right)^{P}$ is assumed to be $\left(\boldsymbol{p}_{n+1}^{I}\right)^{P}=\boldsymbol{p}_{n}^{I}$. Both curves are compared with the monolithic procedure, which solution has been evaluated using a full Newton-Raphson procedure until convergence has been reached. All of those solutions have been obtained with an identical time step interval.

From the plots in Fig. 4, we conclude that the prediction step defined in (70) introduces a significant improvement 
Table 5 Staggered time integration scheme using a predictor step
LOOP over time steps: $(n+1)$

(i) Prediction:

$$
\left(\boldsymbol{p}_{n+1}^{I}\right)^{P}=\boldsymbol{p}_{n}^{I}+\Delta \boldsymbol{p}^{I} \frac{\Delta t_{n+1}}{\Delta t_{n}} \quad \forall I=1, \ldots, n_{f} ;
$$

Initialize:

$$
\begin{aligned}
& \left(p_{n+1}^{I}\right)^{(0)}=\left(p_{n+1}^{I}\right)^{P} \\
& \left(p_{n+1}^{I}\right)^{(-1)}=\left(p_{n}^{I}\right) ; \\
& \left(q_{n+1}\right)^{(0)}=q_{n} ;
\end{aligned}
$$

WHILE NOT CONVERGED: iteration $k$

(ii) Solve nodal displacements: Given $\left(\boldsymbol{q}_{n+1}^{(k-1)}, \boldsymbol{p}_{n+1}^{I^{(k-1)}}\right)$

$$
\text { Compute: } \boldsymbol{K}_{\boldsymbol{u} \boldsymbol{u}} ; \boldsymbol{K}_{\boldsymbol{u} \beta \boldsymbol{I}} ; \boldsymbol{R}_{\boldsymbol{u}} \text { and: }
$$

$$
\boldsymbol{q}_{n+1}^{(k)}=\boldsymbol{q}_{n+1}^{(k-1)}+\left(\boldsymbol{K}_{u u}\right)^{-1}\left(-\boldsymbol{R}_{\boldsymbol{u}}-\sum_{I=1}^{n_{f}} \boldsymbol{K}_{u \beta^{I}}\left(\boldsymbol{p}_{n+1}^{I^{(k-1)}}-\boldsymbol{p}_{n+1}^{I^{(k-2)}}\right)\right)
$$

(iii) Solve nodal slip displacements: Given $\left(\boldsymbol{q}_{n+1}^{(k)}, \boldsymbol{p}_{n+1}^{I^{(k-1)}}\right)$

$$
\text { DO: } I=1, \ldots, n_{f} \quad \text { (loop on fibers) }
$$$$
\text { Compute: } \mathbb{K}_{\beta^{I} \beta^{I}} ; \mathbb{K}_{\beta^{I} u} ; \mathbb{R}_{\beta^{I}} \text { and: }
$$

$$
p_{n+1}^{I^{(k)}}=p_{n+1}^{I^{(k-1)}}+\left(\boldsymbol{K}_{\beta^{I} \beta^{I}}\right)^{-1}\left(-\boldsymbol{R}_{\beta^{I}}-\boldsymbol{K}_{\beta^{I} u}\left(q_{n+1}^{(k)}-q_{n+1}^{(k-1)}\right)\right)
$$

END DO (loop on fibers)

\section{END WHILE}
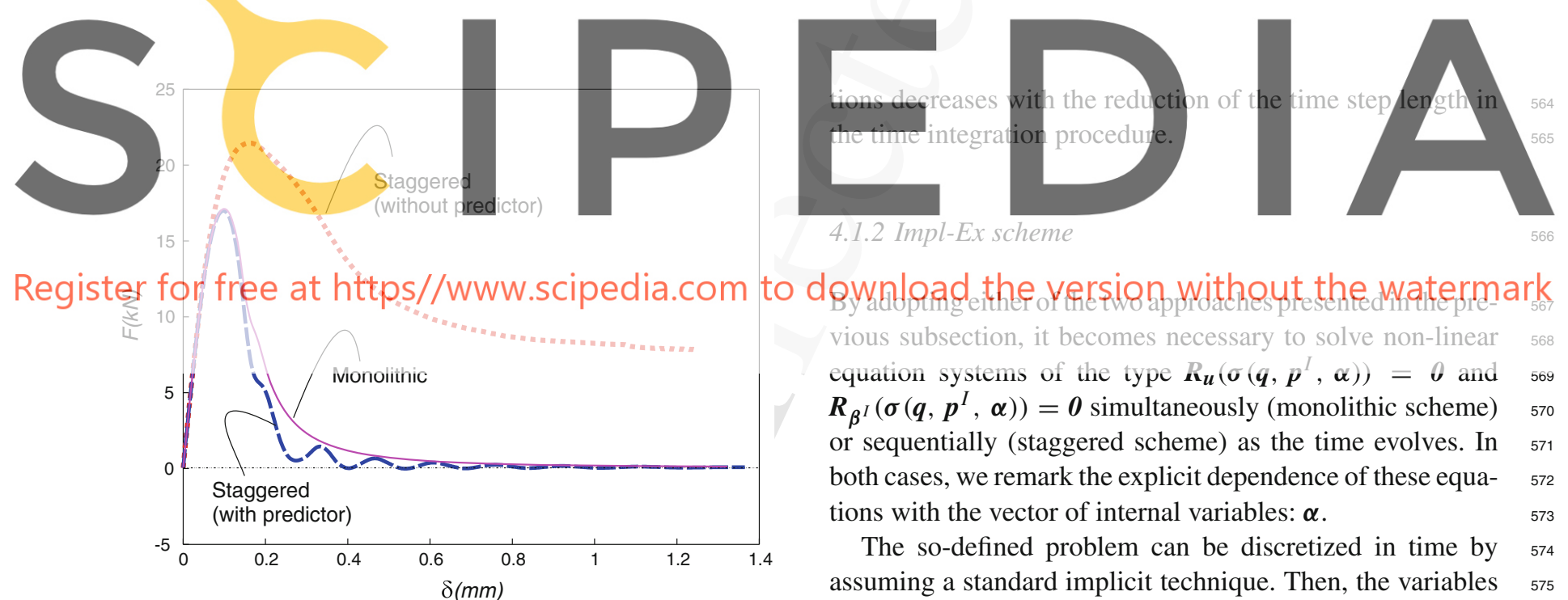

4.1.2 Impl-Ex scheme

d sywalpad the version witheut the watermark vious subsection, it becomes necessary to solve non-linear equation systems of the type $\boldsymbol{R}_{\boldsymbol{u}}\left(\sigma\left(\boldsymbol{q}, \boldsymbol{p}^{I}, \boldsymbol{\alpha}\right)\right)=0$ and $\boldsymbol{R}_{\boldsymbol{\beta}^{I}}\left(\boldsymbol{\sigma}\left(\boldsymbol{q}, \boldsymbol{p}^{I}, \boldsymbol{\alpha}\right)\right)=\boldsymbol{0}$ simultaneously (monolithic scheme) or sequentially (staggered scheme) as the time evolves. In both cases, we remark the explicit dependence of these equations with the vector of internal variables: $\boldsymbol{\alpha}$.

The so-defined problem can be discretized in time by assuming a standard implicit technique. Then, the variables at step $(n+1), \boldsymbol{q}_{n+1}, \boldsymbol{p}_{n+1}^{I} \boldsymbol{\alpha}_{n+1}$ and $\boldsymbol{\sigma}_{n+1}$, must be solved, typically by means of a Newton-Raphson scheme.

However, it is well known that, when dealing with material failure problems, the nonlinear equation systems resulting from a fully implicit discretization methodology show a marked lack of robustness.

In Oliver et al. [15] and [14] an alternative algorithm, the so called Impl-Ex algorithm, has been presented to reduce the nonlinearity of the resulting equations without losing the stability of the computed solution, which is very convenient because it demands a very reduced computational cost. Here, we describe a summary of this methodology that can be easily adapted for modeling HPFRC composite.
568 569 570 571 572 573 574 575 576 577 578 579 580 581 582 583 584 585 586 587 588 
At the time step $(n+1)$, the internal variables of the model are evaluated through two integration procedures:

(i) an implicit standard procedure, which determines, from Tables 1, 2 and 3, $\boldsymbol{\alpha}_{n+1}$ and $\boldsymbol{\sigma}_{n+1}$;

(ii) a predictor (explicit) procedure, here called Impl-Ex variable and denoted with the symbol (*), such as follows:

$$
\tilde{\boldsymbol{\alpha}}_{n+1}=\boldsymbol{\alpha}_{n}+\Delta \tilde{\boldsymbol{\alpha}}_{n+1}
$$

$$
\Delta \tilde{\boldsymbol{\alpha}}_{n+1}=\left(\boldsymbol{\alpha}_{n}-\boldsymbol{\alpha}_{n-1}\right) \frac{\Delta t_{n+1}}{\Delta t_{n}} \text {. }
$$

After replacing these Impl-Ex internal variables into the constitutive equations, Tables 1,2 and 3 , the incremental (rate) stress term, $\Delta \tilde{\boldsymbol{\sigma}}_{n+1}$, is determined from these equations, and the Impl-Ex stress at time $n+1$ is given by:

$$
\tilde{\boldsymbol{\sigma}}_{n+1}=\sigma_{n}+\Delta \tilde{\boldsymbol{\sigma}}_{n+1} .
$$

The Eqs. (62) and (63) are then solved with the Impl-Ex stresses $\tilde{\boldsymbol{\sigma}}_{n+1}$ :

$\boldsymbol{R}_{\boldsymbol{u}}\left(\tilde{\boldsymbol{\sigma}}_{n+1}\right)=\mathbf{0}$

$\boldsymbol{R}_{\boldsymbol{\beta}^{I}}\left(\tilde{\boldsymbol{\sigma}}_{n+1}\right)=\boldsymbol{0}$.

It can be shown, see Oliver et al. [14], that, even during the material softening regime, the consistent tangent matrices, arising from this integration algorithm, are constant (during a time step) and positive definite. As a result of this property, only one iteration per time step is required to get convergence when the solution of Eq. (78) are searched through a NewtonRaphson procedure.

Summarizing, the combination of: (i) a staggered scheme with the prediction stage of the previous subsection and removing global iterations for convergence, plus, (ii) the Impl-Ex procedure for solving each partition of the equation systems; defines a very robust algorithm for solving problems involving HPFRC composites with arbitrary distribution of fibers, which results in a very efficient methodology.

\subsection{Concrete fracture model}

The loss of the linear mechanical response in HPFRC composites depends on the crack phenomena happening in the cementitious component and its interaction with fibers through the fiber-matrix bond. Establishing a satisfactory constitutive model of a HPFRC composite material displaying failure, then requires a concrete crack model that is strongly coupled with the fiber-matrix bond-slip mechanism.

It is known that local constitutive models with strain softening, such as the damage model presented in Table 1, leads to theoretical and numerical difficulties which reflect into spurious numerical solutions. The goal of a well-posed numerical simulation tool is then to adopt a methodology providing objective results respect to the finite element mesh, avoiding the typical mesh size and bias dependence.

In the present approach, the mesh size dependence is removed through the regularization of the softening model of concrete. We reach this objective by introducing a model characteristic length related to the finite element mesh size and the fracture energy of the component. Thus, the softening modulus $H_{m}$ in Table 1 is redefined, and replaced in the table by the intrinsic softening modulus defined by: $\bar{H}_{m}=-\left(G_{f} E_{m} /\left(\sigma_{m}^{u t}\right)^{2}\right) h^{e}$, where $G_{f}$ is the concrete fracture energy, $E_{m}$ and $\sigma_{m}^{u t}$ have been defined in Table 1 and $h^{e}$ is a representative finite element size consistent with the crack orientation (see additional details in [13]).

As for removing the spurious mesh orientation dependence, constants strain localization modes are injected, via a mixed finite element formulation, such as proposed by Dias et al. [5] and Dias [4], and summarized in the following subsection.

\subsubsection{Strain injection method for computational modeling of material failure}

Let us consider standard irreducible quadrilateral finite elements, which are defined as the underlying elements. It is well-known the flaws that this classical element shows for capturing and simulating evolution of cracks.

In order to remove these flaws, we adopt a technique that is mathematically consistent, based on a mixed (assumed strain) variational formulation. This procedure is adopted because it has been shown that mixed formulations, in general, have much better abilities to capture and propagate localizations modes if compared to irreducible formulations.

Assumed strain mixed formulation: the injection domain Let us consider the material bifurcation analysis that is based on detecting the singularity of the acoustic tensor:

$\operatorname{det}\left(\left[\boldsymbol{n} \cdot \mathbb{C}\left(t_{B}\right) \cdot \boldsymbol{n}\right]\right)=0$,

where $\mathbb{C}\left(t_{B}\right)$ is the constitutive tangent tensor of the overall response given by Eq. (45). Equation (79) provides the bifurcation time $t_{B}$, as well as, the normal vector $\boldsymbol{n}$ to the possible crack surface. The numerical resolution of the discontinuous material bifurcation problem has been solved in an effective and accurate way, using a numerical algorithm, based on the iterative resolution of a coupled eigenvalue problem in terms of the localization tensor. This algorithm has been presented in Oliver et al. [17].

After the criterion (79) has been satisfied in a given finite element, we equip the element with an assumed strain model 
that is formulated in the context of a mixed two-field $\left(\overline{\boldsymbol{u}}-\boldsymbol{\varepsilon}_{m}\right)$ variational approach. The interpolated displacement field remains the same as that of the irreducible quadrilateral finite element model presented at the beginning of this section, see Eq. (49). While, the strain field, $\boldsymbol{\varepsilon}_{m}$, is interpolated with functions taken from $\mathcal{V}_{\varepsilon}$, where $\mathcal{V}_{\varepsilon}$ is the space of element-wise constant functions. Then, strains $\boldsymbol{\varepsilon}_{m}$ are associated with displacements through the following variational equation:

$\int_{\mathcal{B}}\left(\boldsymbol{\varepsilon}_{m}-\nabla^{s} \dot{\bar{u}}\right): \delta \boldsymbol{\varepsilon} d \mathcal{B}=\boldsymbol{0} ; \quad \forall \delta \boldsymbol{\varepsilon} \in \mathcal{V}_{\varepsilon}$

From where, the strain matrix (53) in the element $e$ can be written as:

$\left[\boldsymbol{\varepsilon}_{m}\right]^{e}=\overline{\boldsymbol{B}}^{e} \boldsymbol{q}^{e}$

$\overline{\boldsymbol{B}}^{e}=\frac{1}{\mathcal{B}^{e}} \int_{\mathcal{B}^{e}} \boldsymbol{B}^{e} d \mathcal{B}^{e}=\mathbf{0}$,

and Eq. (59) is consequently evaluated by using the modified strain-displacement matrix $\overline{\boldsymbol{B}}^{e}$ instead of $\boldsymbol{B}^{e}$.

The variational equilibrium expression (47), of Table 4 , is rewritten as follows:

$\int_{\mathcal{B}} \boldsymbol{\sigma}\left(\boldsymbol{\varepsilon}^{e}\right): \delta \boldsymbol{\varepsilon} d \mathcal{B}-\int_{\mathcal{B}} \boldsymbol{b} \cdot \delta \boldsymbol{u} d \mathcal{B}-\int_{\partial \mathcal{B}_{\sigma}} \boldsymbol{t}^{*} \cdot \delta \boldsymbol{u} d S=0 ;$

$\forall \delta \boldsymbol{u} \in \mathcal{V}_{0} ; \quad \forall \delta \boldsymbol{\varepsilon} \in \mathcal{V}_{\varepsilon}$

and after replacing the interpolation of displacement and strain fields and changing the matrix $\boldsymbol{B}^{e}$ by: $\overline{\boldsymbol{B}}^{e}$, this equation can be identically written to the expression (62), (63).

The domain where the constant strain mode is injected, is defined as the geometrical locus of the points satisfying:

$\mathcal{B}_{i n j}(t):=\left\{\boldsymbol{X} \in \mathcal{B} \mid t \geq t_{B}(\boldsymbol{X}) ; \dot{r}_{m}(\boldsymbol{X}, t)>0\right\}$,

where the last condition $\left(\dot{r}_{m}(\boldsymbol{X}, t)>0\right)$ means that the concrete component of the composite should be evolving in a loading condition. It is a well known fact that the Assumed Strain Mixed formulation, given by (80) and (81), is unstable if it is applied to the entire discrete domain. Then, it is important to inject the mixed formulation only in the reduced domain of the finite elements satisfying (83) (Fig. 5).

For the numerical implementation of the injection procedure, it is selected the four node quadrilateral element with the standard four Gauss points with one additional Gauss points, placed in the central point of the element.

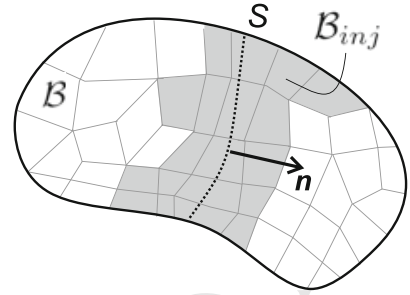

Fig. 5 Strain injection domain

\section{Assessments of the numerical model}

In order to ascertain the suitability of the proposed formulation for describing the structural response of the composite, a selected set of experimental results is taken from the literature. Elastic, hardening and localization stages are examined.

The HPFRC composite model should reproduce two relevant and influential mechanisms, namely, the fiber pullout phenomenon and the subsequent fiber plasticity. In order to show these model features, some tests are particularly addressed in the following sections.

Physical observations of the HPFRC composite behavior show that their failure modes primarily depends on the distribution, content and type of fibers within the specimen. In the next numerical simulations, we show that the model predicts reasonably well the expected failure modes of HPFRC composite with different contents of fibers.

The main concern in this section is to examine: (1) the validation of the model, as well as, its predictive ability, (2) evaluation of the injection procedure in order to improve the finite element mesh-bias dependence during the strain localization process, (3) the effect that the fiber-matrix interface has on the failure mode description and the structural performance.

In the following three cases, we adopt fibers having diameters equal to: $3 \mathrm{~mm}$. Then, the ratio $\Pi / A=1.33 \mathrm{~mm}^{-1}$. Also, in all cases we have taken a residual bond strength: $\tau_{\Gamma}^{R}=0 \mathrm{MPa}$.

\subsection{Notched strip under uniaxial loading}

A notched strip (in plane strain) undergoing uniaxial loading is simulated. The strip and loading conditions are shown in Fig. 6a. It is clamped at its left end and pulled at the right end. The notches are situated in the middle of the specimen to ensure damage localization in this area. The region of concern is the area close to the notch, where the pullout process is expected.

In this test, the complex interaction between the mesoscopic phenomenon such as: the cement fracture, the fibermatrix debonding and the fiber plasticity, can be more easily comprehended and evaluated. 

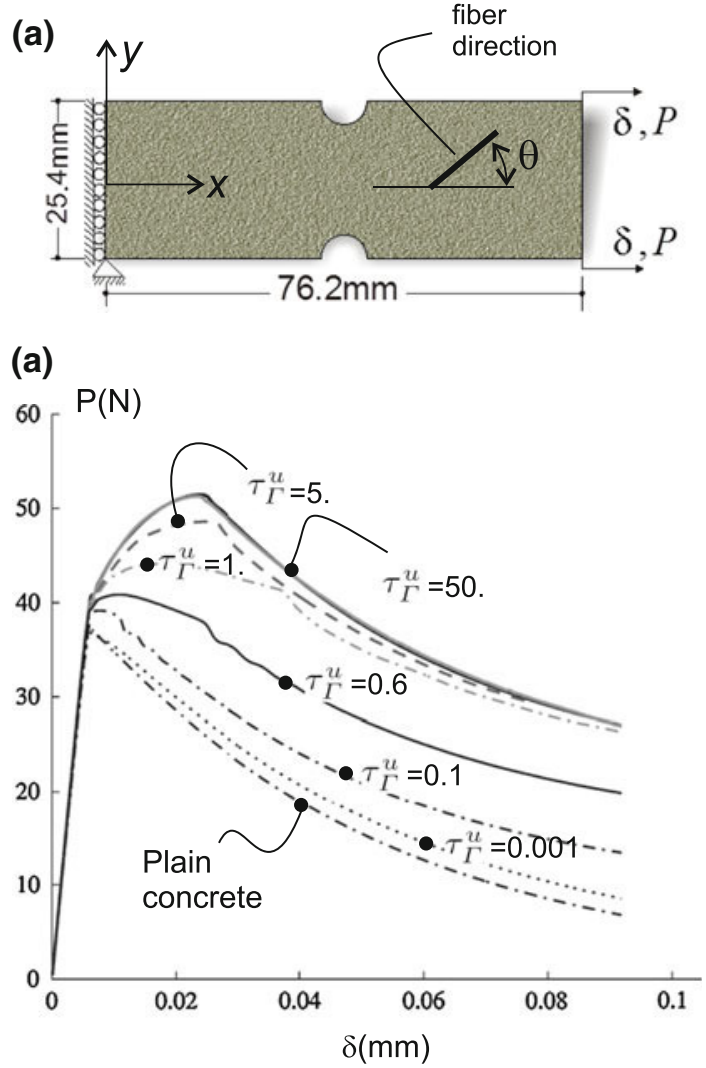

Fig. 6 Notched strip under uniaxial loading: a test setup, b comparison between load versus displacement curves using different ultimate bond shear stresses

The fiber pullout mechanism is analyzed when the fibers are parallel to the principal stretch direction.

\subsubsection{Tensile behavior of the specimen with aligned steel fibers}

Numerical simulations with identical mechanical and geometrical characteristics are carried out, but varying the bond properties of the fiber-matrix interface. The set of parameters is summarized in Table 6. In order to investigate the sensitivity of the model with the ultimate matrix-fiber bond shear strength, $\tau_{\Gamma}^{u}$, six different values of this parameter are considered. While, only one horizontally oriented fiber bundle is assumed $\left(\theta=0^{\circ}\right)$.

Figure 6b compares the load $P$ versus displacement $\delta$ response of the specimen for the six values $\tau_{\Gamma}^{u}$. Included in the plots are the structural response of the plain concrete.

The ascending behavior of the responses are characterized, as we will explain later, by bonded, or partially debonded, matrix-fiber interfaces. As it may be surmised, the hardening behavior is related to the debonding process.

To understand these numerical results, we recall from experimental tests that the HPFRC composites, in tension,
Table 6 Material parameters of the notched specimen under uniaxial loading

\begin{tabular}{lll}
\hline Matrix & Fiber & Bond (fiber-matrix) \\
\hline$\sigma_{m}^{u t}=2.0 \mathrm{MPa}$ & $\sigma_{f}^{y}=210 \mathrm{MPa}$ & $\tau_{\Gamma}^{u}=0.001,0.1, \ldots$ \\
& & $\ldots 0.6,1,5,50 \mathrm{MPa}$ \\
$E_{m}=15.0 \mathrm{GPa}$ & $E_{f}=200 \mathrm{GPa}$ & $G_{\Gamma}=1 . e 8 \mathrm{GPa}$ \\
$v_{m}=0.2$ & $H_{f}=0 \mathrm{MPa}$ & $H_{\Gamma}=100 \mathrm{MPa}$ \\
$G_{f}=100 \mathrm{~N} / \mathrm{m}$ & $\theta=0^{\circ}$ & $k_{f}=0.75 \%$ \\
\hline
\end{tabular}

displays three stages: linear elastic (that ends when the first crack in the specimen arises), multicrack or hardening stage (that ends at the peak point), and the strain localization stage. Also we recall that, in the tensile load-displacement response, the main difference between the HPFRC composite and the conventional FRC is the multicrack stage after finalizing the linear response. The multicrack stage may not exist in the conventional FRC.

The response for the smallest value of the ultimate bond shear stress considered in this example, $\tau_{\Gamma}^{u}=0.001 \mathrm{MPa}$, closely resembles the curve displayed by the plain concrete case. As expected, the numerical results show brittle behavior for the plain concrete material. After the peak load has been reached, the material softens and ductility is barely evidenced.

The load-displacement curves for increasing values of $\tau_{\Gamma}^{u}$ display increasing hardening, as well as, increasing peak load values. However, with $\tau_{\Gamma}^{u}>5 \mathrm{MPa}$, the response of the material no longer change significantly. Then, in the present specific problem, we could assert that $\tau_{\Gamma}^{u}=5 \mathrm{MPa}$ represents a limit bond strength.

Figure 7 depicts the iso-color maps of damage distribution in cement at the end of analysis. Different cases, depending on $\tau_{\Gamma}^{u}$, are shown. Figure 7a displays the tendency of the plain concrete response showing a highly concentrated damage pattern. With increasing $\tau_{\Gamma}^{u}$, according with Fig. 7b:f, the zone affected by damage grows, suggesting that an increasing number of fibers are subjected to the pullout effect, and, in consequence, the material toughness increases.

Analysis of the interaction effects between matrix, fiber and interface debonding For identical time steps, sequential portraits of plasticity in fibers, matrix damage and matrix-fiber interface debonding distributions can be superimposed to visualize the failure characteristics of each compound. The analysis, at the microstructural level, reveals various failure mechanisms which synergistic interaction accounts for the larger strength and higher toughness properties. The analysis is performed with four values of $\tau_{\Gamma}^{u}=[0.001,1,5,50 \mathrm{MPa}$. In concordance with these values, we distinguish three different failure mechanisms, depending on the fiber-matrix bond responses: (i) fully debonded fibers, (ii) partially debonded 


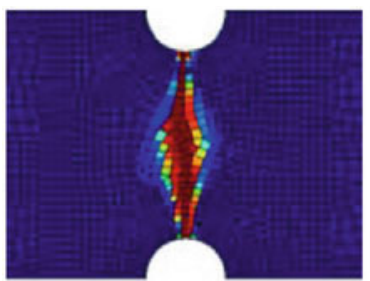

Plain concrete

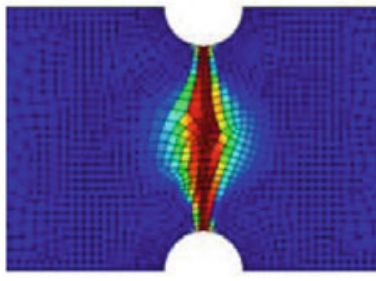

$\tau_{\Gamma}^{u}=0.1$

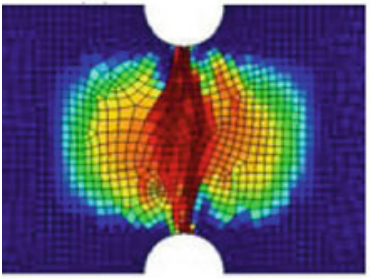

$\tau_{\Gamma}^{u}=1$

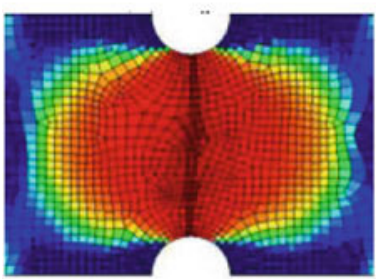

$\tau_{\Gamma}^{u}=50$

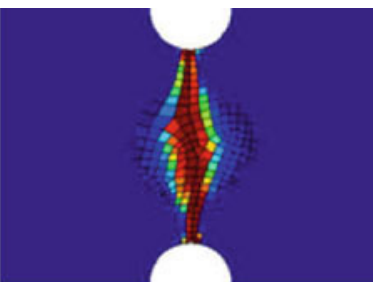

$\tau_{\Gamma}^{u}=0.001$

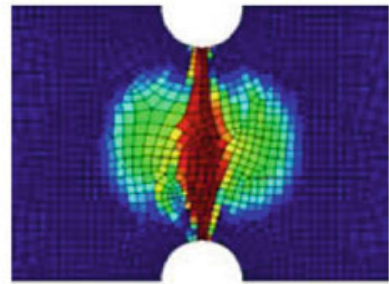

$\tau_{\Gamma}^{u}=0.6$

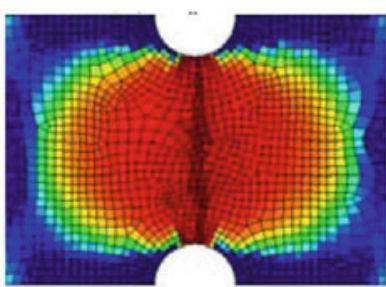

$\tau_{\Gamma}^{u}=5$

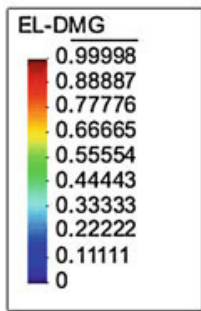

Fig. 7 Damage distribution in cement matrix with different matrixfiber bond strength parameters fibers and (iiii) fully bonded fibers. They are specifically analyzed in the following items.

\section{(i) Fully debonded fibers: $\left(\tau_{\Gamma}^{u}=0.001 \mathrm{MPa}\right)$}

Weak fiber-matrix interfaces are generally associated to a low fracture toughness of the composite. A weak interface posses low fiber-matrix stress transfer capacity and, therefore, the fiber strengths are not fully utilized. According to the results in Fig. $6 \mathrm{~b}$, low ductility is associated with $\tau_{\Gamma}^{u}=0.001 \mathrm{MPa}$. Under tensile loads, the model shows a sudden debonding in the whole domain, as it is observed in the debonding distribution of Fig. 8 with a consequent loss of the material composite effect. In this case, we verify that for enough small values of the ultimate bond strengths, the model is able to represent weak fiber-matrix interfaces. In fact, this (a)

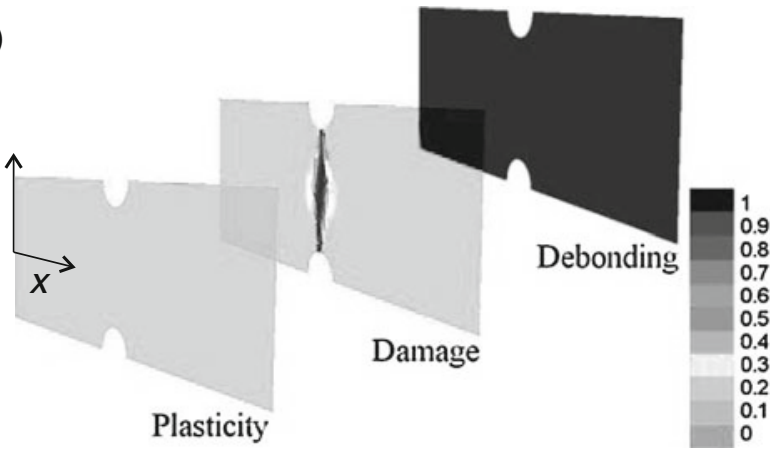

(b)

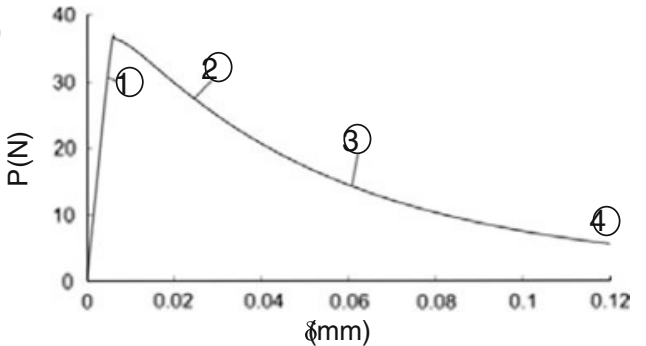

(c)

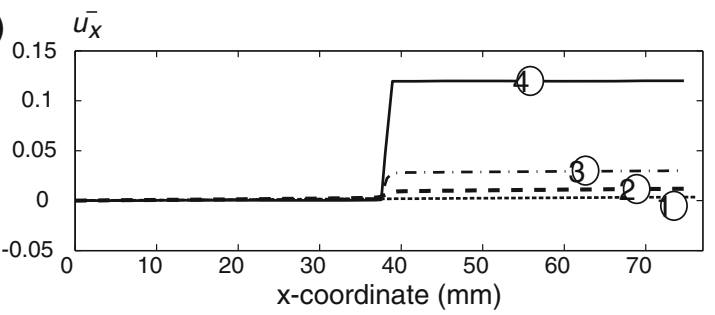

(d)

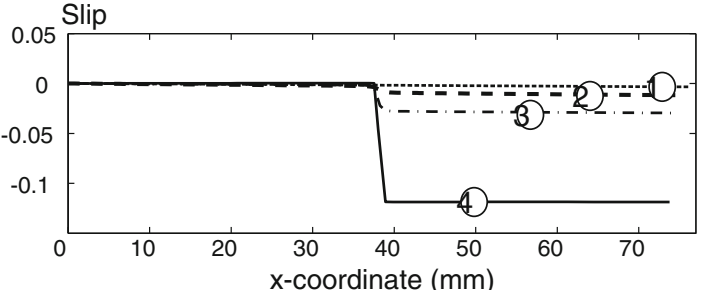

Fig. 8 Results for $\tau_{\Gamma}^{u}=0.001 \mathrm{MPa}$. a Distribution portraits of fiber plasticity, fiber-matrix interface debonding and matrix damage at the end of analysis. The fiber-matrix interface debonding map is represented with only two states: 0 is no-debonding $\left(\tau_{\Gamma}<\tau_{\Gamma}^{u}\right), 1$ is debonding meaning that in some loading stage $\left(\tau_{\Gamma}=\tau_{\Gamma}^{u}\right)$. Damage map ranges between 0 and 1 , b load versus displacement curve, $\mathbf{c} \bar{u}_{x}$ displacement plot along the specimen horizontal direction (numbers are in correspondence with the loading stages shown in $\mathbf{b}$ ), $\mathbf{d}$ plots of the fiber-matrix slip $\beta$ along the specimen horizontal direction (numbers are in correspondence with the loading stages shown in $\mathbf{b}$ )

is a consequence of the Capriz balance equation, which governs the microstructural behavior. When using $\tau_{\Gamma}^{u} \approx 0 \mathrm{MPa}$, the fiber strain also approaches to zero, and therefore, the fiber is pulled out from the matrix immediately after the load is applied. Also, this implies that the slip $\beta$ can take any arbitrary value after the bond strength is exhausted. Certainly, the value of the slip is of the same order of magnitude than the displacement $\bar{u}_{x}$, as shown in Fig. 8c, d, where the $\operatorname{slip} \beta$ and $\bar{u}_{x}$ displacement are plotted along the length of the strip 


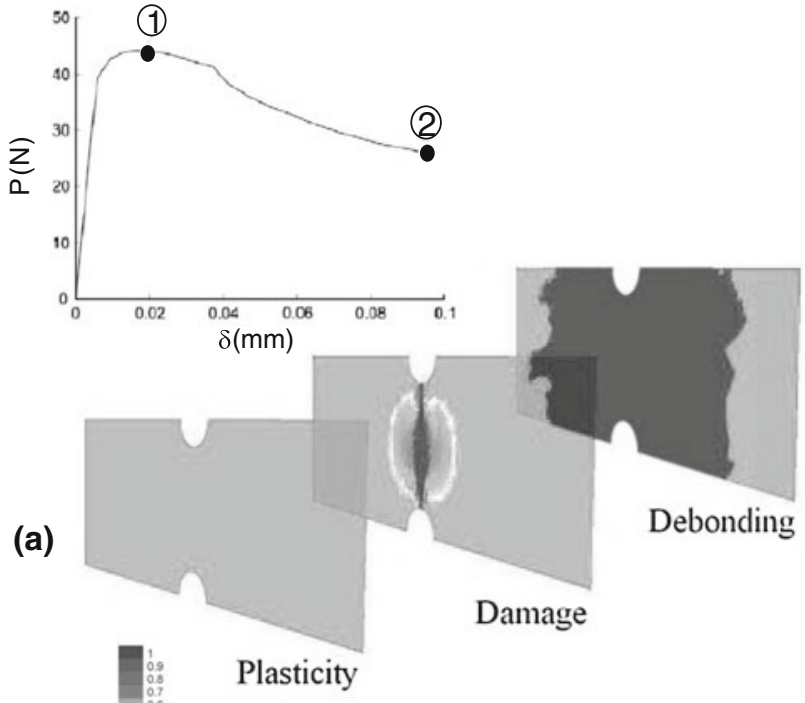

(b)

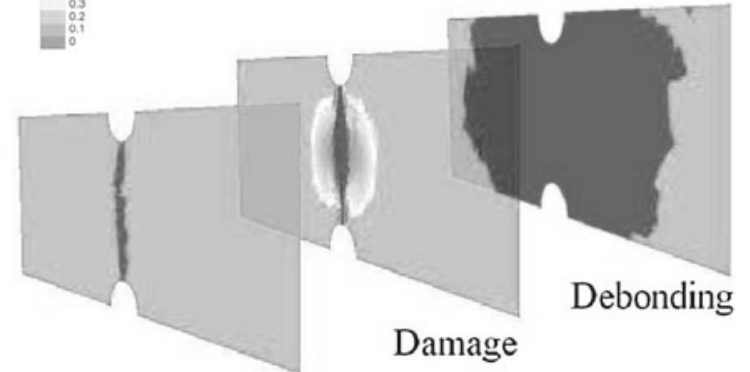

Plasticity

Fig. 9 Results for $\tau_{\Gamma}^{u}=1 \mathrm{MPa}$. Distribution portraits of fiber plasticity, fiber-matrix interface debonding and matrix damage at the end of analysis: a stage 1 at the softening regime onset. b Stage 2 in the end of the loading process

in different stages of the loading curve as indicated in Fig. 6b. Damage concentration in the notch section is due to the inability of the fiber-matrix interface to transfer the stresses. According with the damage and debonding results in Fig. 7, small axial strain in the fibers is developed due to the sudden debonding, and consequently, yielding is not achieved, as confirmed in the fiber plasticity distribution.

(ii) Partially debonded fibers: $\left(\tau_{\Gamma}^{u}=1 \mathrm{MPa}\right)$

In the case simulated with $\tau_{\Gamma}^{u}=1 \mathrm{MPa}$, which in accordance with the Fig. 6 displays semi-ductile behavior, represents a partially debonded example. The results obtained in this case are shown in two different instants indicated in Fig. 9. The first instant, Fig. 9a, represents a stage during the hardening process. The second instant, Fig. 9b, represents a stage at the end of the localization process.

The assumed perfect plastic material behavior adopted for the matrix-fiber bond slip relationship gives rise to the slip when the ultimate bond shear strength is reached, and subsequently the shear deformation is increased. In the first instant, depicted in Fig. 9a, it is noticeable that fiber-matrix interface

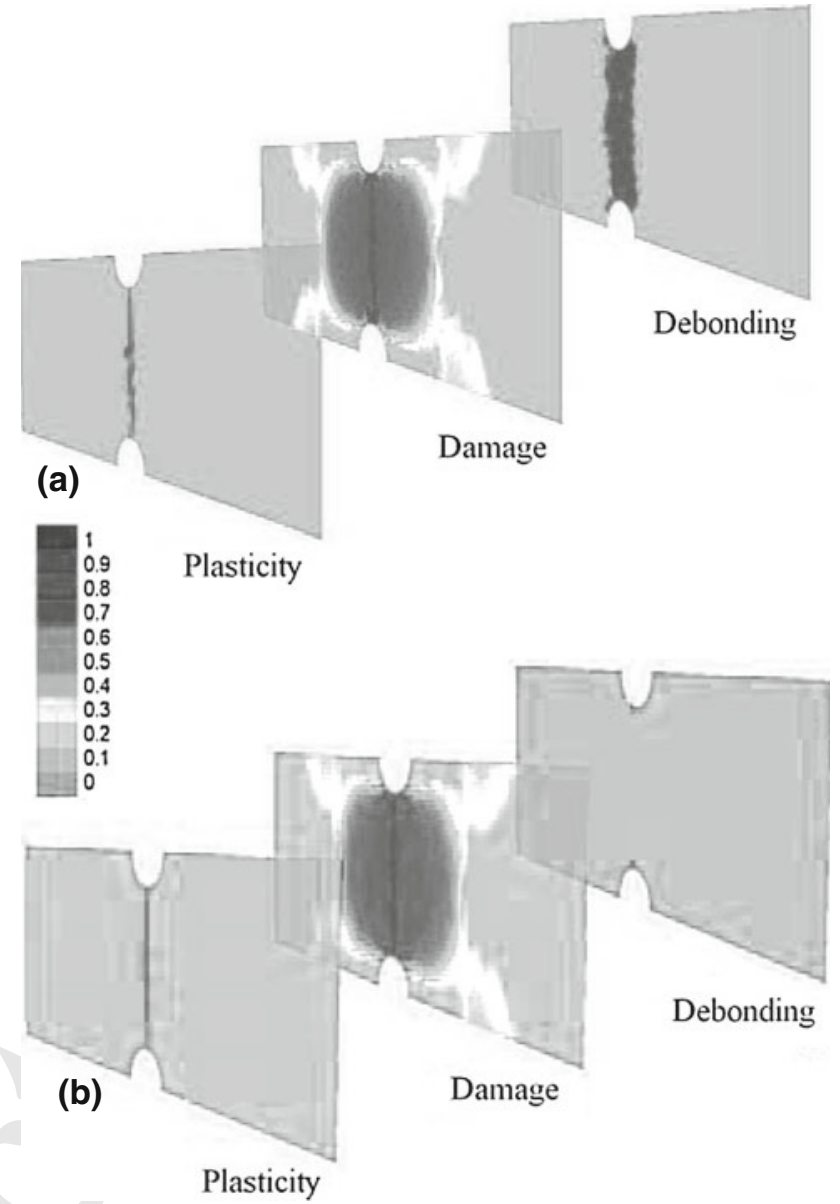

Fig. 10 Distribution portraits of fiber plasticity, fiber-matrix interface debonding and matrix damage at the end of analysis: a results for $\tau_{\Gamma}^{u}=$ $5 \mathrm{MPa}$. b Results for $\tau_{\Gamma}^{u}=50 \mathrm{MPa}$. Scales of the iso-colour maps for the plasticity, damage and debonding distributions are similar to the description given in the legend of Fig. 9

debonding evolves as a consequence of the increase in the slip. Fiber-matrix interface debonding and matrix damage may be triggered because of their weakness to resist shear stresses. This behavior indicates that the matrix damage and sliding frictional resistance of fiber pullout largely determine the composite toughness and the hardening properties (Beyerlein and Phoenix [2]). Inspection of the plots for damage and plasticity in the second stage, Fig. 9b, indicates that the crack opening in the notch, due to cumulative damage, is accompanied with loss of adhesive bond in the matrix-fiber interface and plastic strain in fibers.

After comparing the debonding maps of stage 2 in Fig. 9 with those of stage 1 , we note that only few more points in the specimen achieve the ultimate bond shear strength at the end of analysis.

(iiii) Fully bonded fibers to the matrix: $\left(\tau_{\Gamma}^{u}=5-\right.$ $-50 \mathrm{MPa})$

High adhesive interfaces can be achieved by improving, at microstructural level, the properties of fiber surface. 

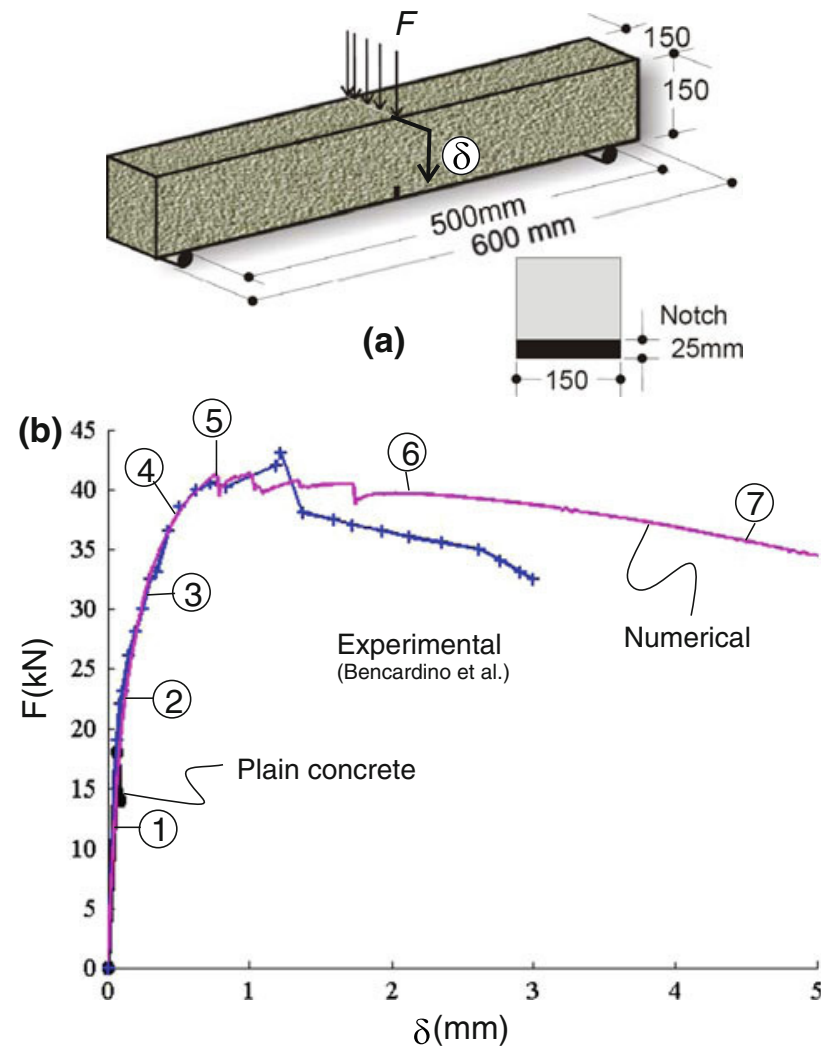

(c)

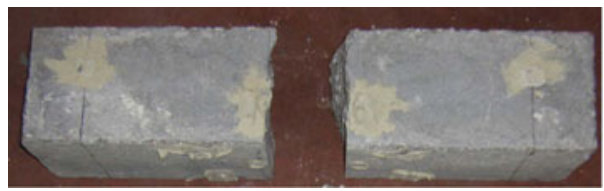

Fig. 11 Notched three point beam bending test with randomly distributed fibers. a Specimen geometry, b numerical and experimental load $F$ versus vertical displacement $\delta$ of the loading application point in three point notched beam test

Table 7 Material parameters adopted in the model to simulate the notched beam specimen test under flexural loading

\begin{tabular}{lll}
\hline Matrix & Fiber & Bond (fiber-matrix) \\
\hline$\sigma_{m}^{u c}=21.25 \mathrm{MPa}$ & $\sigma_{f}^{y}=2100 \mathrm{MPa}$ & $\tau_{\Gamma}^{u}=5.1 \mathrm{MPa}$ \\
$E_{m}=13.89 \mathrm{GPa}$ & $E_{f}=210 \mathrm{GPa}$ & $G_{\Gamma}=1 . e 8 \mathrm{GPa}$ \\
$v_{m}=0.2$ & $H_{f}=100 \mathrm{MPa}$ & $H_{\Gamma}=100 \mathrm{MPa}$ \\
$G_{f}=100 \mathrm{~N} / \mathrm{m}$ & $\theta=\left[0^{\circ}, 10^{\circ}, 20^{\circ}\right.$, & $k_{f}=1 \%$ \\
& $30^{\circ}, 45^{\circ}, 60^{\circ}$, & \\
& $\left.70^{\circ}, 80^{\circ}, 90^{\circ}\right]$ & \\
\hline
\end{tabular}

However, a strong interface may result in lower toughness, because this effect does not allow interfacial debonding, which is one of the main mechanisms to relieve stress concentrations produced by the oncoming crack (Jiang et al. [7]). With a view towards investigating this possibility, simulations were performed for $\tau_{\Gamma}^{u}=5$ and $50 \mathrm{MPa}$.

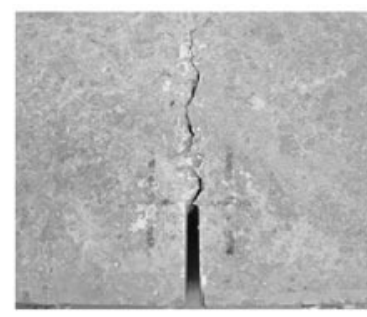

(a)

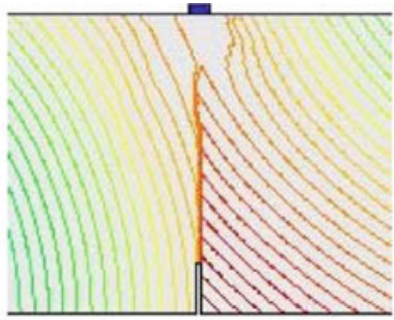

(b)

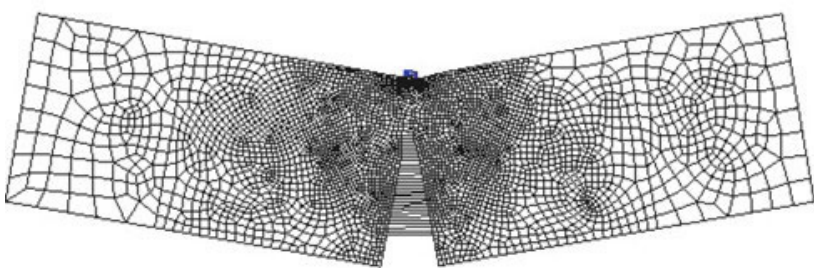

(c)

Fig. 12 Experimental and numerical crack pattern in the three point notched beam test. a Experimental, b numerical, $\mathbf{c}$ deformed finite element mesh

Observing the corresponding load-displacement curves in Fig. 6b, the model predict similar structural responses. Even more remarkable is the fact that plasticity and damage exhibit similar distribution patterns at the end of the loading process. It seems reasonable to propose, based on these results, that in both situations, the dominant failure mechanism at the mesoscale is the fiber plasticity. Although the debonding distribution is distinctively different, this effect does not seem to affect significantly the structural response. Experimental results corroborate that if the fiber-matrix interface strength is much higher than the matrix strength in shear, then, the matrix damage will occur instead of fiber-matrix debonding. This experimental fact is also supported through numerical simulation by observing results for $\tau_{\Gamma}^{u}=50 \mathrm{MPa}$ in Fig. 10 . Although the matrix multicracking is much more significant in this case, plastic deformation in fibers occurs in the path of the critical crack. In summary, the pullout process, and in consequence the failure mechanism for high adhesives interfaces, involves essentially matrix damage and plasticity.

\subsection{Notched three point beam bending test with a random distribution of fibers}

The numerical analysis of degradation mechanisms in beams bending tests, built of HPFRC composites with fiber oriented in only one direction, such as that presented in Oliver et al. [18], are illustrative because the results of these kind of tests are simpler to interpret. Nevertheless, reinforced composites with randomly orientated fibers are much more frequent in practical cases.

The present numerical simulation is addressed to analyze a notched beam with randomly distributed short fibers. The 

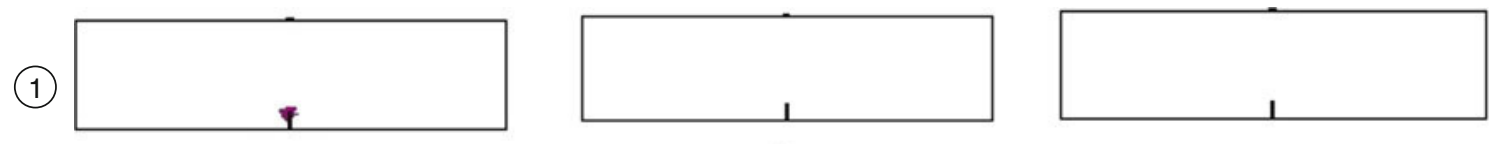

(3)

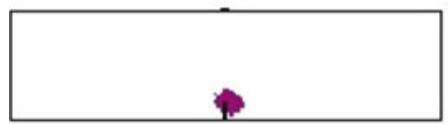

(4)

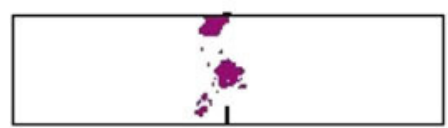

(6)

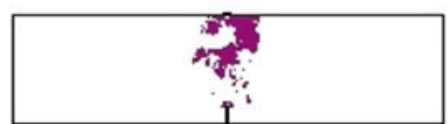

(7)

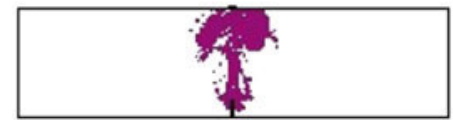

(a) $0^{\circ}$

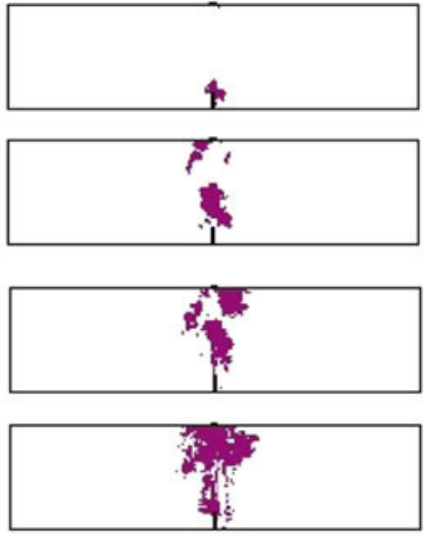

(b) $45^{\circ}$
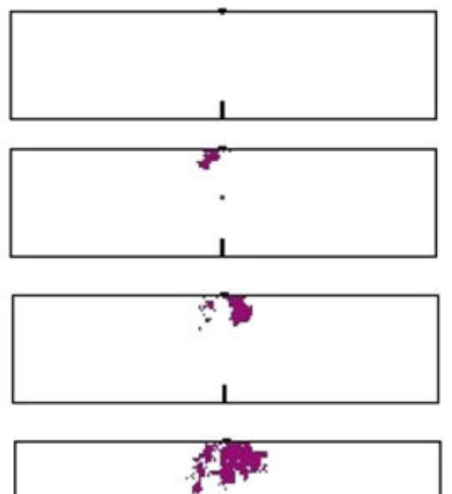

(c) $90^{\circ}$

Fig. 13 Evolution of the matrix-fiber debonding process in the notched beam test for three fiber bundles directed along $0^{\circ}, 45^{\circ}$ and $90^{\circ}$ respect to the horizontal direction. Stages 1, 3, 4, 6 and 7 correspond to the points marked in Fig. 11b

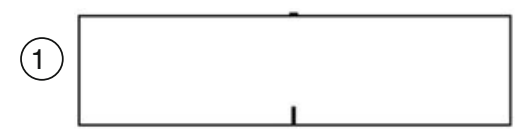

(3)

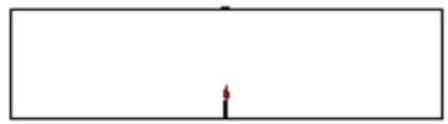

(4)

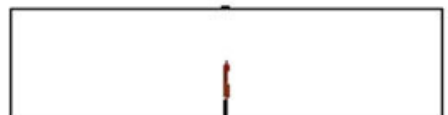

(6)

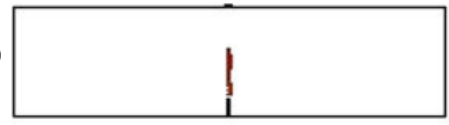

(7)

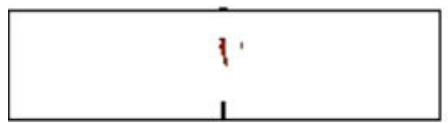

(a) $0^{\circ}$
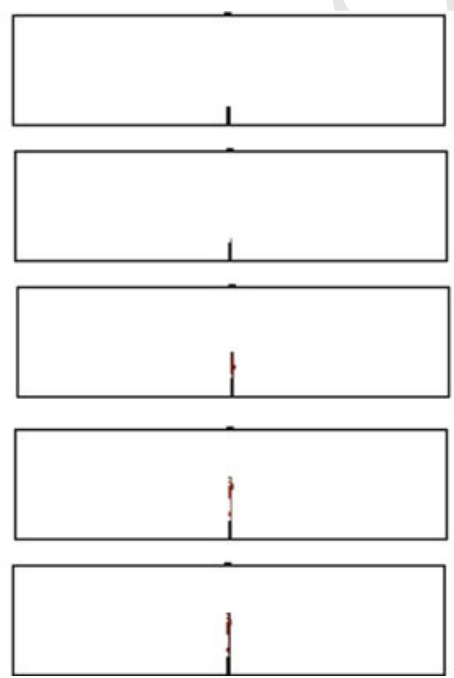

(b) $45^{\circ}$
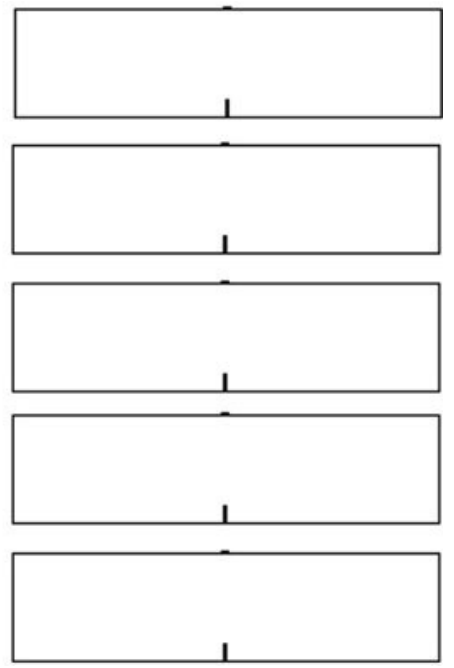

(c) $90^{\circ}$

Fig. 14 Evolution of fiber plasticity in three point notched beam test for three fiber bundles directed along $0^{\circ}, 45^{\circ}$ and $90^{\circ}$ respect to the horizontal direction. Stages 1, 3, 4, 6 and 7 correspond to the points marked in Fig. 11b

experimental test corresponding to this case has been presented by Bencardino et al. [1], and has been carried out according to the RILEM specification [19]. The beam geometry is shown in Fig. 11a.

In Table 7, we define the mechanical properties adopted in the numerical model for the matrix, fiber bundles and fibermatrix interface. Also, we assumes that nine fiber bundles represent sufficiently well the random distribution of fibers. The finite element model, assumed as a plane stress condition, consists of 3,938 quadrilaterals with 4,032 nodes.

The experimental load versus displacement curve, of a FRC specimen with fiber fraction volume equal to $1 \%$, is presented in Fig. 11b, (taken from Bencardino et al. [1]). In the same plot, we compare the numerical solution. Experimental and numerical curves agree quite well up to the peak load. However, after this point, the numerical model slightly overestimates the postcritical response. Also, in the same plot, the experimental unreinforced (plain concrete) specimen is shown. A brittle behavior is observed.

\subsubsection{Mesostructural behaviour}

In the experimental test, a complete separation of the specimens into two parts has occurred, as shown in Fig. 11c. The 
(1)

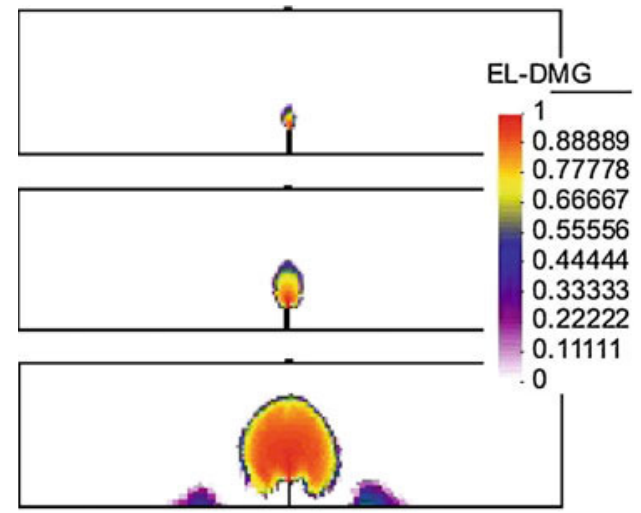

(4)

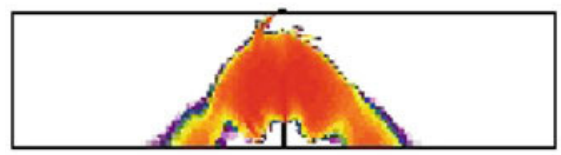

(5)

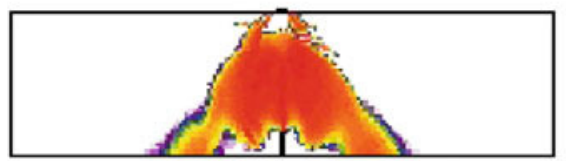

(7)

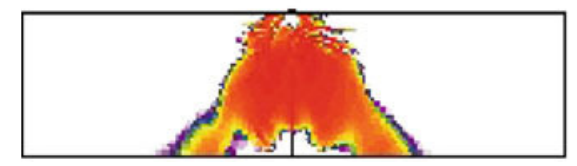

Fig. 15 Damage evolution in three point notched beam test
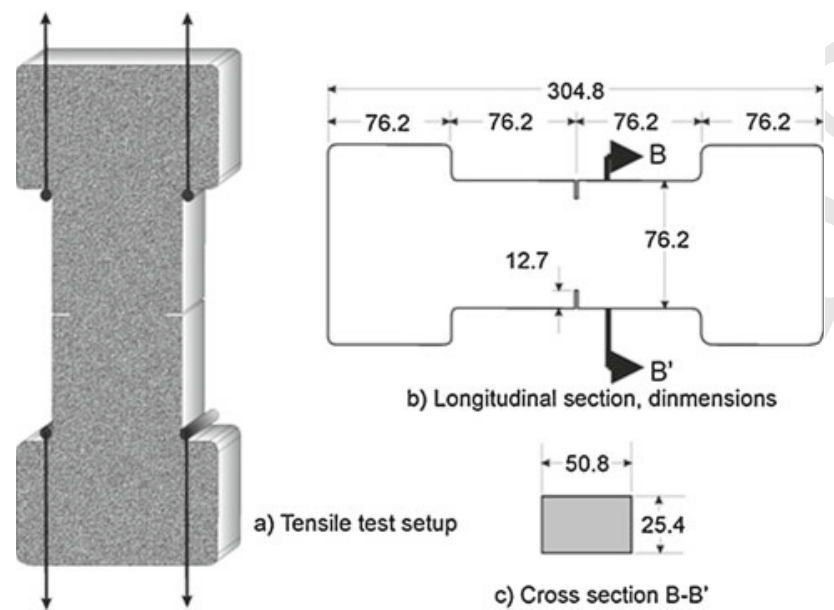

b) Longitudinal section, dinmensions

$+50.8+$

a) Tensile test setup

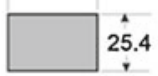

c) Cross section B-B'

Fig. 16 Double-notched dogbone specimen tensile test (Suwannakarn [21])

finite element simulation also predicts a single crack, see Fig. 12a:b. However, the specimen does not split abruptly in two parts as for the unreinforced beam. The deformed configuration of the beam after loading is scaled by 10 in Fig. 12c.

Figures 13 and 14 display the evolution of the simultaneous capacity loss of matrix-fiber bound, as well as, the plastic strains of fibers, respectively. Three bundles of fibers

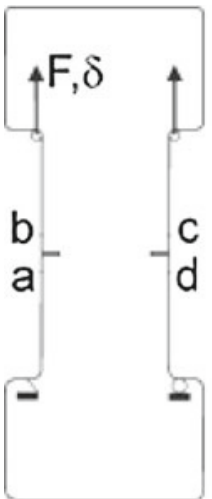

(a)

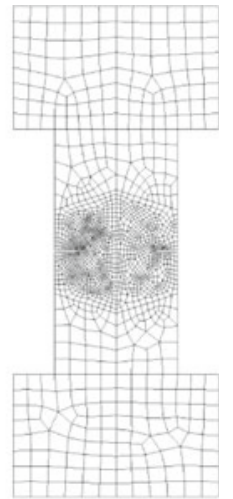

(b)
Fig. 17 Double-notched dogbone specimen tensile test: a test layout, b finite element mesh

Table 8 Material parameters

\begin{tabular}{lll}
\hline Matrix & Fiber & Bond (fiber-matrix) \\
\hline$\sigma_{m}^{u t}=1.25 \mathrm{MPa}$ & $\sigma_{f}^{y}=2100 \mathrm{MPa}$ & $\tau_{\Gamma}^{u}=5.1 \mathrm{MPa}$ \\
$E_{m}=13.89 \mathrm{GPa}$ & $E_{f}=210 \mathrm{GPa}$ & $G_{\Gamma}=1 . e 8 \mathrm{GPa}$ \\
$v_{m}=0.2$ & $H_{f}=100 \mathrm{MPa}$ & $H_{\Gamma}=100 \mathrm{MPa}$ \\
$G_{f}=100 \mathrm{~N} / \mathrm{m}$ & $\theta=\left[0^{\circ}, 10^{\circ}\right.$, & $k_{f}=0.75 \%$ \\
& $20^{\circ}, 30^{\circ}, 45^{\circ}$, & \\
& $60^{\circ}, 70^{\circ}, 80^{\circ}$, & \\
& $\left.90^{\circ}\right]$ & \\
&
\end{tabular}

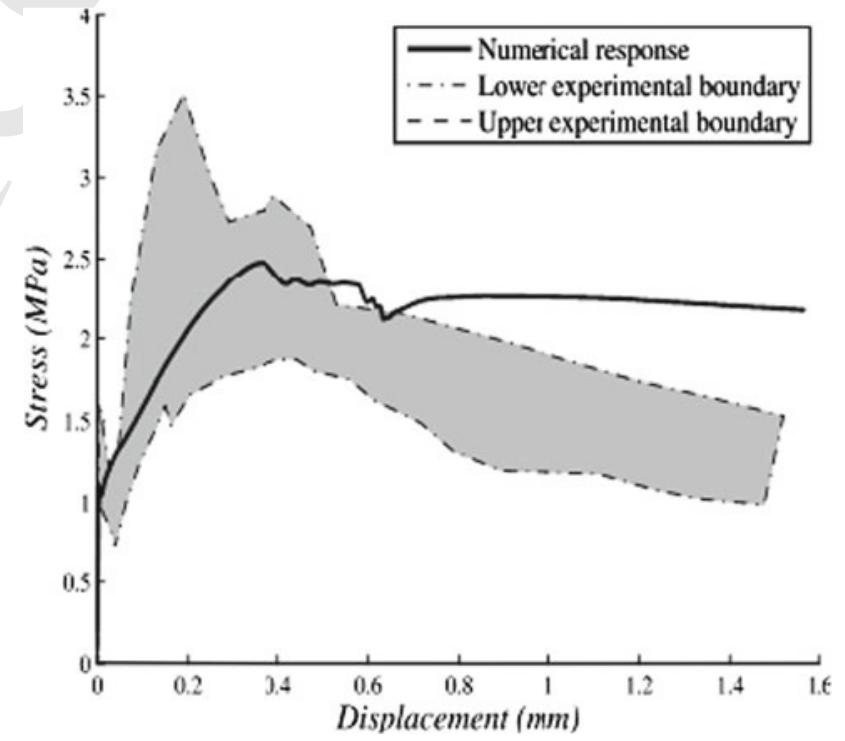

Fig. 18 Numerical and experimental structural response in double notched dogbone test. Average stress versus $\delta$ displacement. (a) Numerical. (b) Experimental (Suwannakarn [21])

(oriented to $0^{\circ}, 45^{\circ}$ and $90^{\circ}$ ) and different stages along the load deflection curve are specifically analyzed. According to these results, the evolution of both mechanisms are con- 
(a)

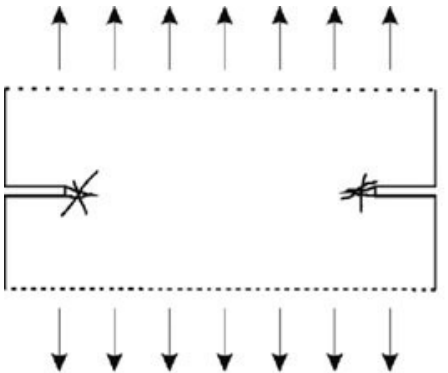

(b)

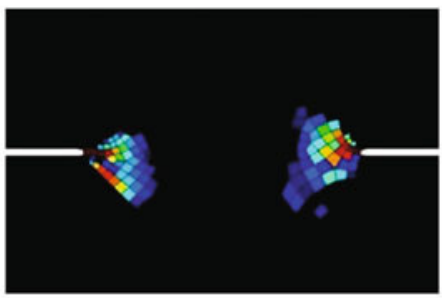

(c)

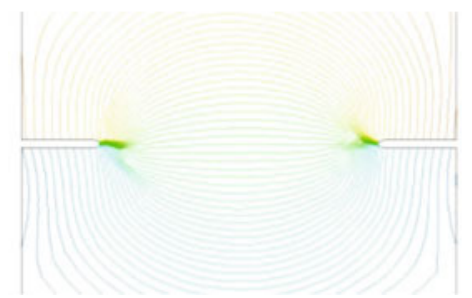

(1)
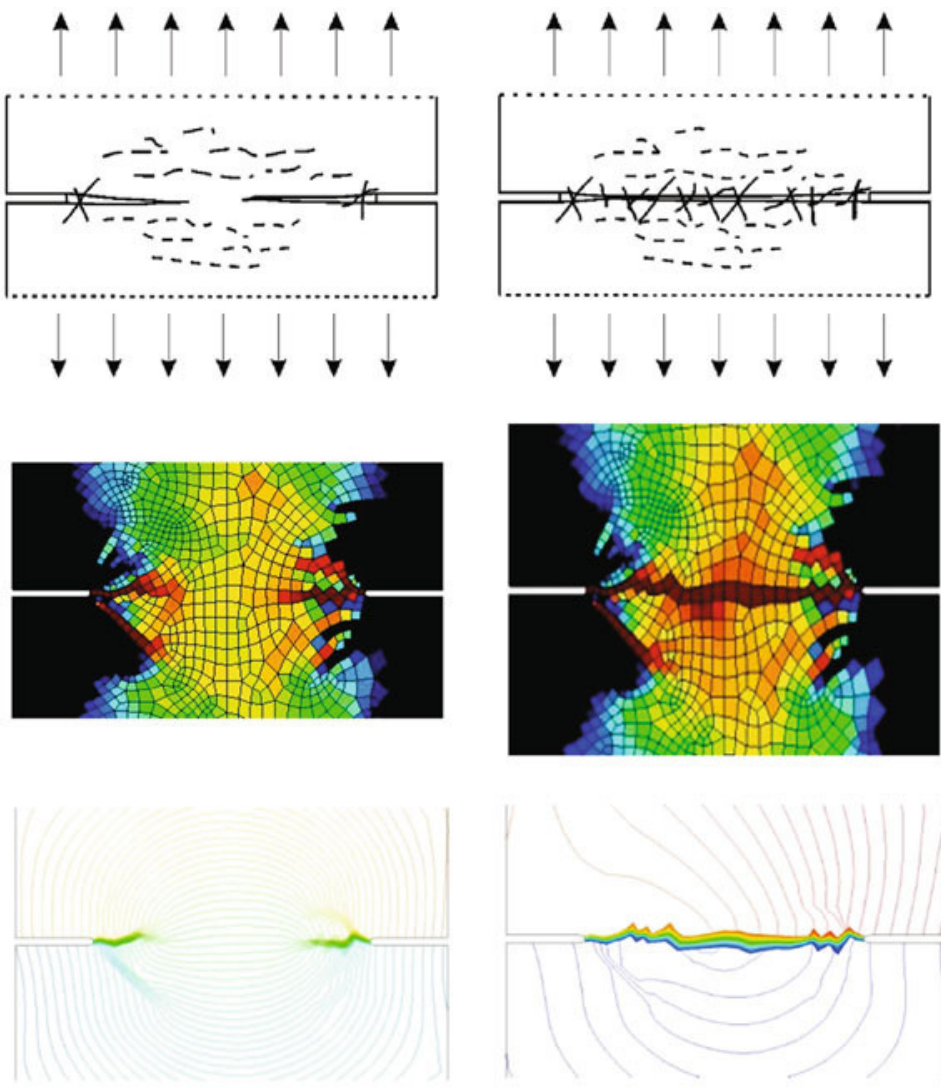

(2)

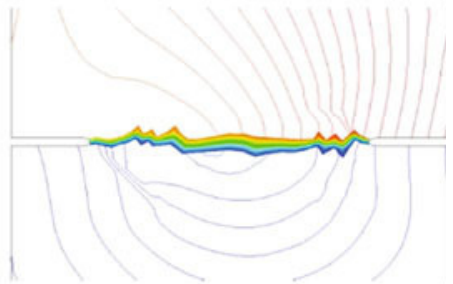

(3)

Fig. 19 Tensile response in double notched test: a typical crack propagation and strain localization in HPFRC composites as described in Suwannakarn [21], b damage distribution ( $d \geq 0.98)$, $\mathbf{c}$ iso-displacement curves displaying the macrocrack formation and evolution

centrated in the region near the notch, where crack propagation is expected to occur. The attention is addressed initially to analyze the debonding distribution of the fiber bundles oriented $0^{\circ}$ and $45^{\circ}$ respect to the horizontal direction (Fig. 13a, b, respectively). These results suggest that the loss of the adhesion in the interface zone starts during the initial loading stages. However, for the bundle fiber oriented $90^{\circ}$, Fig. $13 \mathrm{c}$, the distribution displays that the process begins later and it does not affect the area located near the notch.

\subsubsection{Damage evolution and localization process}

Microcracking in the cement matrix occurs simultaneously with debonding and plasticity of fibers during the fracture process. Figure 15 displays the iso-color damage maps in six different stages that are identified in the load versus displacement plot of Fig. 11b.

In the stages 1 and 2, few elements around the notch are damaged. As loading progresses, the damage region spreads over beyond the notch section. In the stage 3 , some elements in the bottom part of the beam begin to damage. From stage
3 , the damaged region covers the middle third and remains almost unaltered until the end of the loading process. Darker red color stands for completed damage material. According to the iso-color map in the stage 7, severe degraded material is presented in the notch proximity. However, comparing this result and the iso-displacement contours in Fig. 12b only a single vertical macrocrack, initiated in the notch root, is developed.

\subsection{Double-notched dogbone specimen tensile test}

According with Suwannakarn [21], from where we take the experimental results of this test, the dogbone-shaped notched specimen is well adapted to control the location of the crack position. To ensure an adequate propagation path, the specimen has symmetrical notches at their mid section. Additionally, this test setup is useful to measure the composite fracture properties of HPFRC composites and estimate the size of a pseudo-plastic zone which corresponds to the cracked area of the matrix.

The geometric details of the specimen are shown in Fig. 16. Dimensions are given in Fig. 16b, c for the longitudinal 
(a)

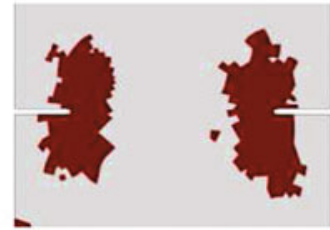

$\theta=0^{\circ}$

(b)

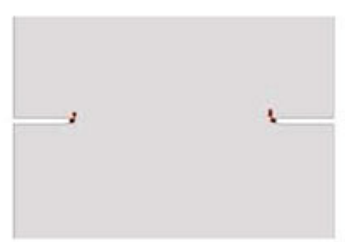

(c)

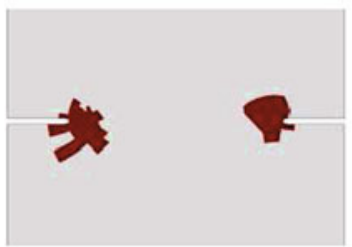

$\theta=90^{\circ}$

(d)

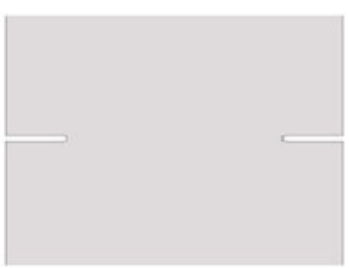

(1)

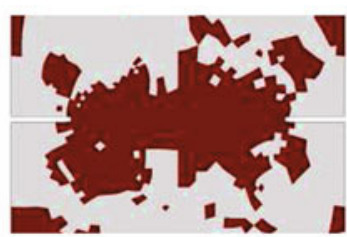

Debonding

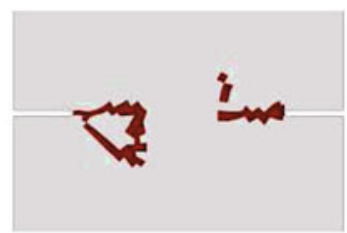

Plasticity

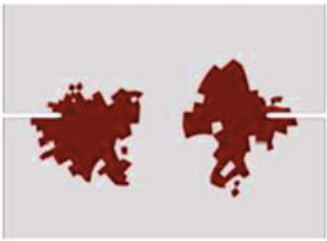

Debonding

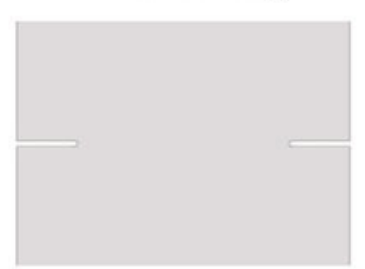

Plasticity

(2)
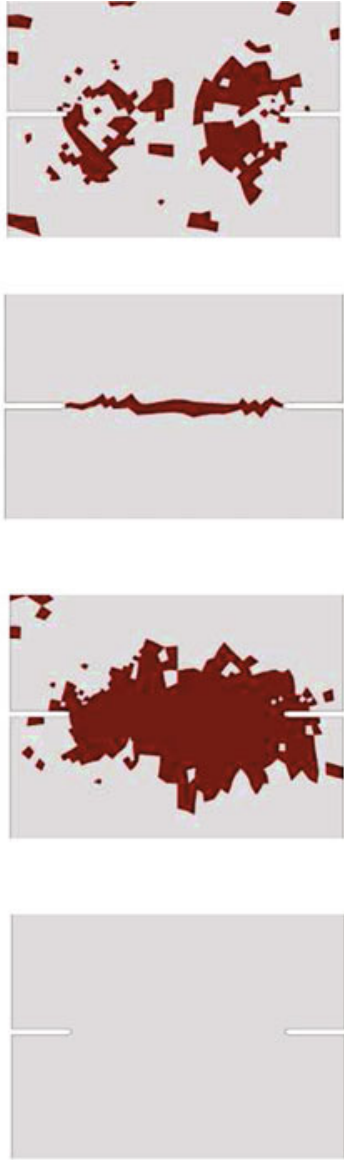

(3)

Fig. 20 Main stages of debonding and plasticity evolution of the loading process in the dogbone test. a Debonding distribution (fiber bundle: $\left.\theta=0^{\circ}\right)$. b Plasticity distribution $\left(\theta=0^{\circ}\right)$. c Debonding distribution (fiber bundle: $\theta=90^{\circ}$ ). $\mathbf{d}$ Plasticity distribution $\left(\theta=90^{\circ}\right.$ )

and cross section, respectively. The loading process consists of imposing displacements at the specimen top, while fixing the bottom, as indicated in Fig. 17a.

The material parameters for this example are summarized in Table 8. The specimen is reinforced with hooked end fibers and has a fiber volume fraction of $0.75 \%$. The test is simulated using 1,639 quadrilateral finite elements in plane stress condition. In order to capture the concrete fracture phenomena, we adopt the formulation presented in the previous section based on the injection of constant strain localized modes. The finite element mesh is shown in Fig. 17b.

Figure 18 plots the stresses versus crack opening displacements obtained with the numerical simulation. This result is compared with the envelope of the experimental tests which were obtained with a small number of specimens (only 3 ). In both, numerical and experimental results, can be observed that after the initial elastic response, the structural behavior displays a hardening effect even when a multiple crack phenomena is developing. The numerical result follows this trend and lies within the experimental envelopes. However, after crossing the peak load value, the numerical solution deviates from the experimental data.

\subsubsection{Crack propagation analysis in the notched hooked end fiber specimen}

In Fig. 19, we analyze the crack propagation phenomenon. The numerical solution displays a fracture process starting in the roots of the specimen notches, such as observed by Suwannakarn. And cracks evolve in a similar manner to that described in Fig. 19a.

There are three main stages represented in Fig. 19 corresponding to: the onset of the first crack (column 1 of pictures), multiple cracks, associated with strain hardening (column 2), and (3) the strain localization stage, associated with strain softening (column 3).

As the specimen is subjected to tensile loads, damage concentration arises mostly at the notch root proximity, as it is shown in the first stage of Fig. 19b. After initiation, the damage extends in several directions (stage 2 of Fig. 19b). A large 
Fig. 21 Failure mode in the double notched test. a Numerical. b Experimental (Suwannakarn [21])

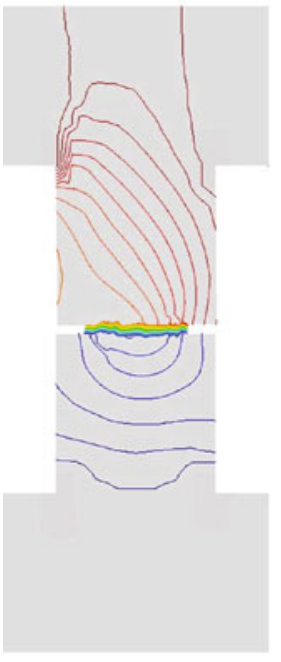

(a)

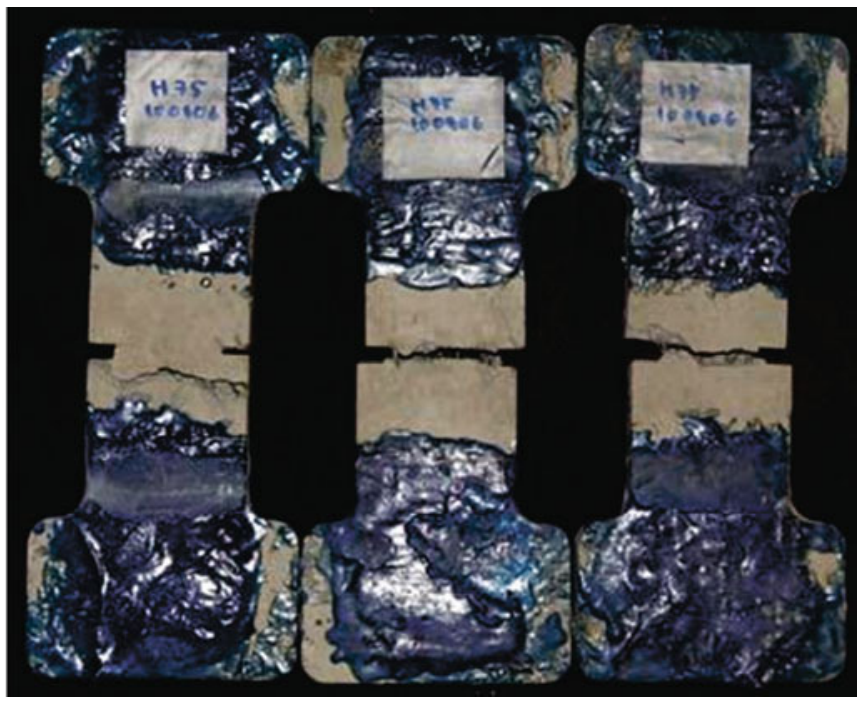

(b) damage zone is observed during this stage, but the macrocracks formed at the notch roots do not propagate, probably arrested by the fibers. According to Suwannakarn, the first crack does not necessarily propagates across the section, prior to the formation of other cracks. The numerical results have captured this effect.

Figure 19c shows iso-displacement contours plot in the three considered stages. As can be seen in the contour plots, the macrocrack propagates from the notch roots to the specimen center, nonetheless, branching at the notches is observed in the initial stages, so that more than one crack are competing.

Pictures in Fig. 20 compares the debonding and plastic evolution in the fiber bundles oriented at angles: $\theta=0^{\circ}$ and $90^{\circ}$ respect to the loading direction. Observing Fig. 20b, $\mathrm{c}$, they suggest that the pullout process involves initially, a debonding action, which provides several alternative paths for the crack propagation. And second, fiber plastic deformation, which contributes directly to the total deformation of the composite. According with these results, the debonding action is preceded by the formation of new surfaces at the fiber-matrix interface; therefore, reduction of composite strength may be significantly related to the loss of interface resistance. Figure 20d also reveals that plastic deformation is not observed in horizontally placed fiber bundles. However, debonding plots in Fig. 20c predict loss of adhesive and frictional capacity in the interface zone.

Iso-displacement contours for total displacements $(\mid \overline{\boldsymbol{u}} \|)$, in Fig. 21a, shows a single macrocrack between the two symmetrical notches. This crack pattern agrees with the experimental observation of the three specimens depicted in Fig. $21 \mathrm{~b}$. The fractured specimens are depicted on the right hand side of the Fig. 21. Since the presence of the notches in the specimen was designed to induce stress concentration in the central region and, in turn, an easy predictability of the crack pattern, nevertheless, experimental findings partially reflect this assertion and show in one case (according to Suwannakarn) a deviated crack path respect to the desired trajectory (Fig. 21b, left).

\section{Conclusions}

Considering the numerical solutions of problems solved in Sect. 5, as well as, those complementing the set of validation tests that were presented in the paper of Oliver et al. [18] using the identical micromorphic model; we conclude that, the numerical approach developed for simulating failure of HPFRC composites is able to capture important effects induced by the concrete fracture and the mechanical interaction between fiber and matrix.

Some parameters that are explicitly considered in the model, and which dramatically affect the composite response leading to the complete failure, are: quantity of fibers, yield strength of fibers and strength of cement. Other important parameters, such as the fiber shape, roughness of the fiber surface, fiber aspect ratio, etc., have been implicitly considered through a phenomenological law describing the shearslip interaction in the fiber-matrix interface. The numerical assessments have highlighted the influence of some of these parameters in the structural responses.

The model introduces as many slip displacement fields, and microscopic balance equations, as fiber bundles are considered in the composite. Therefore, a variable number of dof's per node have to be managed. We have proposed a very efficient numerical procedure to handle this specific feature 
of the problem. It is based on a partitioning of the macro- and microforce balance equations, a predictor step of the slip displacement fields, one for every fiber bundle, and the Impl-Ex integration scheme.

The concrete fracture model included in the numerical procedure uses a technique that is not affected by the finite element mesh [4].

The notched strip results obtained with the simulation model in Sect. 5.1, exemplifies the use of this computational tool. The conclusions obtained from this test, and particularly those obtained in the analysis of the three main cases of bonding in cementitious composites, can be extended to the analysis of more complex situations.

In the tests presented in Sects. 5.2 and 5.3, we have analyzed the effect of fiber plasticity and debonding as a function of the inclination angle of the fiber respect to the load direction. However, there is one mesoscopic effect that the model do not address specifically. In fact, we have assumed that the shear stress-slip law characterizing the mechanical response of the bond, in the fiber-matrix interface, does not changes with the direction of the fiber. According to Lee at al. [8], due to the mechanisms of snubbing, matrix spalling and fiber straightening, these law changes notably with the fiber direction. Future research should be addressed to include this important phenomenon in the numerical model.

Acknowledgements J. Oliver gratefully acknowledges the financial support of the European research Council (ERC) through the 2012- ERC Advanced Grant Project No. 320815. The Spanish Ministry of Science and Innovation, and the Catalan Government Research Department, are also gratefully acknowledged for their financial support to this research under Grants BIA2011-24258 and 2009 SGR 1510, respectively.

\section{References}

1. Bencardino F, Rizzuti L, Spadea G, Swamy RN (2010) Experimental evaluation of fiber reinforced concrete fracture properties. Composites B 41:17-24

2. Beyerlein IJ, Phoenix SL (1996) Stress concentrations around multiple fiber breaks in an elastic matrix with local yielding or debonding using quadratic influence superposition. J Mech Phys Solids 44:1997-2039

3. Capriz G (1989) Continua with microstructure. Springer, Berlin

4. Dias IM (2012) Strain injection techniques in numerical modeling of propagating material failure. $\mathrm{PhD}$ Thesis, Technical University of Catalunya, Barcelona
5. Dias IF, Oliver J, Huespe AE (2011) Strain injection, mixed formulations and strong discontinuities in fracture modeling of quasibrittle materials. In: Proceedings of the congress on numerical methods in engineering 2011, Coimbra, Portugal, 14-17 June, 2011, pp 163-202. APMTAC, LIsbon

6. Frémond M, Nedjar B (1996) Damage, gradient of damage and principle of virtual power. Int J Solids Struct 33:1083-1103

7. Jiang H, Valdez JA, Zhu YT, Beyerlein IJ, Lowe TC (2000) The strength and toughness of cement reinforced with bone-shaped steel wires. Compos Sci Technol 60:1753-1761

8. Lee Y, Kang S, Kim JK (2010) Pullout behaviour of inclined steel fibers in an ultra-high strength cementitious matrix. Constr Build Mater 24:2030-2041

9. Li F, Li Z (2000) Continuum damage mechanics based modeling of fiber reinforced concrete in tension. Int J Solids Struct 36:777-793

10. Linero DL (2006) A model of material failure for reinforced concrete via Continuum Strong Discontinuity Approach and mixing theory. PhD Thesis, E.T.S. Enginyers de Camins, Canals i Ports, Technical University of Catalonia (UPC), Barcelona, 2006. CIMNE Monograph Number, M106

11. Mariano PM (2002) Multifield theories in mechanics of solids. Adv Appl Mech 38:1-93

12. Mariano PM, Stazi F (2005) Computational aspects of the mechanics of complex materials. Arch Comput Methods Eng 12:391-478

13. Oliver J (1989) A consistent characteristic length for smeared cracking models. Int J Numer Methods Eng 28:461-474

14. Oliver J, Huespe AE, Blanco S, Linero DL (2005) Stability and robustness issues in numerical modeling of material failure with the strong discontinuity approach. Comput Methods Appl Mech Eng 195(52):7093-7114

15. Oliver J, Huespe AE, Cante JC (2008a) An implicit/explicit integration schemes to increase computability of non-linear material and contact/friction problems. Comput Methods Appl Mech Eng 197:1865-1889

16. Oliver J, Linero DL, Huespe AE, Manzoli O (2008b) Twodimensional modeling of material failure in reinforced concrete by means of a continuum strong discontinuity approach. Comput Methods Appl Mech Eng 197:332-348

17. Oliver J, Huespe AE, Cante JC, Diaz G (2010) On the numerical resolution of the discontinuous material bifurcation problem. Int $\mathrm{J}$ Numer Methods Eng 83:786-804

18. Oliver J, Mora D, Huespe AE (2012) A micromorphic model for steel fiber reinforced concrete. Int J Solids Struct 49:2990-3007

19. R. T. 162-TDF (2002) Recommendations of RILEM TC 162-TDF: test and design methods for steel fibre reinforced concrete: bending test. Mater Struct 35:579-582

20. Simo J, Hughes T (1998) Computational inelasticity. Springer, Berlin

21. Suwannakarn SW (2009) Post-cracking characteristics of high performance fiber reinforced cementitious composites. $\mathrm{PhD}$ Thesis, University of Michigan 